\title{
Sonoluminescence and bubble dynamics for a single, stable, cavitation bubble
}

\author{
D. Felipe Gaitan, ${ }^{\text {a) }}$ Lawrence A. Crum, Charles C. Church, and Ronald A. Roy \\ National Center for Physical Acoustics, University of Mississippi, Coliseum Drive, \\ University, Mississippi 38677
}

(Received 8 July 1991; revised 28 January 1992; accepted 29 January 1992)

\begin{abstract}
High-amplitude radial pulsations of a single gas bubble in several glycerine and water mixtures have been observed in an acoustic stationary wave system at acoustic pressure amplitudes on the order of $150 \mathrm{kPa}(1.5 \mathrm{~atm})$ at $21-25 \mathrm{kHz}$. Sonoluminescence (SL), a phenomenon generally attributed to the high temperatures generated during the collapse of cavitation bubbles, was observed as short light pulses occurring once every acoustic period. These emissions can be seen to originate at the geometric center of the bubble when observed through a microscope. It was observed that the light emissions occurred simultaneously with the bubble collapse. Using a laser scattering technique, experimental radius-time curves have been obtained which confirm the absence of surface waves, which are expected at pressure amplitudes above $100 \mathrm{kPa}$. [S. Horsburgh, Ph.D. dissertation, University of Mississippi (1990) ]. Also from these radius-time curves, measurements of the pulsation amplitude, the timing of the major bubble collapse, and the number of rebounds were made and compared with several theories. The implications of this research on the current understanding of cavitation related phenomena such as rectified diffusion, surface wave excitation, and sonoluminescence are discussed.
\end{abstract}

PACS numbers: $43.25 . Y w, 43.35 . S x$

\section{INTRODUCTION}

The subject of this paper is the dynamics of bubbles in acoustic cavitation fields of moderate intensities. Acoustic cavitation may be defined as the formation and pulsation of vapor or gas cavities in a liquid under acoustic stress. A particularly interesting phenomenon associated with acoustically driven gas bubbles is the weak emission of light called sonoluminescence (SL). This emission has been attributed to the high temperatures generated during the rapid compression of the bubbles caused by the action of the sound field. Despite the extensive amount of research done on both acoustic cavitation and sonoluminescence, many important questions relating to the nature and dynamics of these phenomena remain unanswered. Attesting to this uncertainty is the multiplicity of existing models describing the mechanisms of light production as well as the number of conflicting views and observations of cavitation-related phenomena found in the literature.

Until very recently, experiments in SL generally involved "clouds" of (sonoluminescing) gas bubbles generated by various types of transducers immersed in different liquids, and driven at high amplitudes. ${ }^{1-3}$ Due to the high amplitudes needed to generate light, the bubbles were made to pulsate nonlinearly and nonradially, causing them to move about rapidly and erratically, and to break up often into smaller parts. ${ }^{4}$ Obviously, the determination of the parameters relevant to SL (bubble radius, driving pressure, internal temperature and pressure, etc.) has been difficult

\footnotetext{
a) Present address: Naval Postgraduate School, Physics Department, Monterey, CA 93943.
}

under these conditions. Researchers therefore have been forced to describe such cavitation systems in terms of ensemble average quantities, or to assume that SL was produced only for a small range of parameter values. In this paper, we report on a recently found region in the driving pressurebubble-radius parameter space in which a single, light-emitting bubble can pulsate radially and stably for an indefinite amount of time. Using this system, the radius of the bubble has been recorded as a function of time and the timing of the SL flashes has been measured relative to the sound field and the bubble radius. The timing of SL has also been used to observe the change of the bubble's equilibrium radius as a function of time.

Cavitation has generally been classified in two types: "transient" and "stable" cavitation. This classification traces its origin to when the first visual observations of cavitation activity were made, ${ }^{5,6}$ and was introduced by Flynn ${ }^{7}$ in order to describe these observations. Transient cavitation was used to describe events that lasted only fractions of a second, usually occurring at high pressure amplitudes. These events were attributed to vapor or gas bubbles that expanded to large sizes during the negative part of the pressure cycle, after which they began to collapse. Because of the large radius attained during the expansion, their collapse was very rapid and violent, often resulting in the destruction of the bubbles. According to Flynn, ${ }^{7}$ a bubble may be represented by a transient cavity "if, on contraction from some maximum size, its initial motion approximates that of a Rayleigh cavity..." or by a stable cavity "if it oscillates nonlinearly about its equilibrium radius." Flynn later $^{8}$ defined the expansion ratio, $R_{\max } / R_{0}$, where $R_{\max }$ is the maximum radi- 
us attained during a given acoustic period and $R_{0}$ is the equilibrium radius, and showed that bubbles pulsating with $R_{\max } / R_{0}$ above this threshold usually developed into transient cavities, whereas bubbles below this threshold developed into stable cavities. Although transient bubbles are not necessarily unstable, they tend to collapse very rapidly, promoting surface instabilities that often result in breakup. Thus, when the transient cavitation threshold is exceeded, bubbles are, in general, not expected to survive for more than a few cycles.

Sonoluminescence (SL) was first observed by Marinesco et al. in $1933^{9}$ when photographic plates submerged in an insonified liquid became exposed. But it was not until 1947 that Paounoff et al. ${ }^{10}$ showed that the exposure of the plates occurred at the locations of pressure maxima (referred to in this paper as "pressure antinodes" or simply "antinodes") of the standing wave field. After several years of investigation, it became clear to researchers that gas bubbles were directly responsible for the generation of light in a sound field.

During the late 1950s and early 1960s, several experiments were done in an attempt to find the relationship between the phase of SL emission and the phase of the sound field. ${ }^{11-15}$ The motivation behind these experiments was to discriminate among the different models based on the conflicting predictions concerning the phase of the SL emission. For example, The Triboluminescence, Microdischarge and Mechanochemical models predict the light to be emitted during bubble growth, whereas the Balloelectric, the Anion Discharge, the Hotspot, and the Chemiluminescence models predict it to occur during the bubble collapse. Although it was not known then, the phase of SL relative to the sound field is dependent on the experimental conditions such as the insonation frequency, the initial bubble radii and the physical parameters of the host liquid ${ }^{16}$ and, for this reason, those experiments were partly unsuccessful.

The general outline of the rest of this paper is as follows: Section I describes briefly three mathematical models used to describe the motion of acoustically driven bubbles. Some of the basic ideas of nonlinear bubble dynamics necessary to understand the experimental results are introduced in this section. Section II contains a detailed description of the apparatus and the experimental arrangements used to acquire the data, including the calibration methods. In Sec. III, the results of the different experiments are presented and discussed, including observations on the stabilization process for a single bubble. Data from the single-bubble experiments are presented first in order to facilitate the interpretation of the multibubble experimental results. Single-bubble data include the radius-versus-time curves and the phase of SL emission. Then, a study of the phase of SL in multibubble cavitation fields is presented. Finally, conclusions drawn from the experiments are summarized in Sec. IV.

\section{THEORY OF BUBBLE DYNAMICS}

\section{A. Introduction}

In this section, we present the mathematical formulations used to describe the motion of a single bubble in a spa- tially uniform acoustic field, which is assumed to vary sinusoidally in time. In the calculations, three different formulations are used, the results of which are compared with the measurements. These formulations are the KellerMiksis ${ }^{17}$ radial equation with a linear polytropic exponent approximation, the Keller-Miksis radial equation with the more exact formulation for the internal pressure due to Prosperetti et al., ${ }^{18}$ and Flynn's ${ }^{19}$ formulation, which also includes thermal effects inside the bubble. These formulations will be called KM-polytropic, KM-Prosperetti, and Flynn formulations, respectively. Because of the large pressure amplitudes $\boldsymbol{P}_{\boldsymbol{A}}$ used in the calculations, only formulations that included correction terms for the compressibility of the liquid were considered. Following the procedure used by Keller and Miksis, ${ }^{17}$ Prosperetti ${ }^{20}$ has obtained the following equation:

$$
\begin{aligned}
\left(1-\frac{\dot{R}}{c}\right) R \ddot{R}+\frac{3}{2} \dot{R}^{2}\left(1-\frac{\dot{R}}{3 c}\right) & \\
= & \left(1+\frac{\dot{R}}{c}\right) \frac{1}{\rho_{l}}\left[p_{B}(R, t)-p_{A}\left(t+\frac{R}{c}\right)-p_{\infty}\right] \\
& +\frac{R d p_{B}(R, t)}{\rho_{l} c d t},
\end{aligned}
$$

where $R$ is the bubble radius, dots indicate time derivatives, $\rho_{l}$ is the liquid density, $c$ is the speed of sound in the liquid, $p_{B}(R, t)$ is the pressure on the liquid side of the bubble interface, and $p_{A}(t+R / c)$ is the time-delayed driving pressure. Here, $p_{B}(R, t)$ can be expressed in terms of the internal pressure by the continuity of normal stress across the boundary

$$
p_{B}(R, t)=p_{g}(R, t)-2 \sigma / R-4 \mu_{l}(\dot{R} / R),
$$

where $p_{g}$ is the gas pressure in the interior of the bubble, $\sigma$ is the air-liquid surface tension, and $\mu_{l}$ is the shear viscosity of the liquid. The relationship between the internal pressure and the bubble radius is determined mainly by the thermodynamic properties of the gas. Although this relation can be assumed to be isothermal or adiabatic under some circumstances, a polytropic relation has often been used ${ }^{7,16,17,21,22}$ of the form

$$
p_{g}=p_{0}\left(R_{0} / R\right)^{3 \kappa} \text {, }
$$

where $\kappa$ is the polytropic exponent, and $p_{0}$ is the internal pressure of the bubble at equilibrium $\left(R=R_{0}\right)$, defined by

$$
p_{0}=p_{\infty}+2 \sigma / R_{0},
$$

where $p_{\infty}$ is the liquid pressure at infinity. The value of $\kappa$ varies between 1 and $\gamma$, for isothermal and adiabatic motion, respectively, $\gamma$ being the ratio of the specific heat capacities $C_{p} / C_{v}$. This exponent is calculated using a method described by Prosperetti. ${ }^{20}$

Although the polytropic approximation has been generally thought to be a fairly accurate model, an experimental study by Crum and Prosperetti ${ }^{23}$ has shown significant discrepancies between theory and experiment for oscillations near the harmonics of the resonance frequency of the bubble. Since compressibility and viscous effects are expected to be small at the amplitudes used by Crum and Prosperetti, their results suggest that thermodynamics plays a more important role in the motion of the bubble than originally expected. As 
a result, a more accurate expression for the internal pressure has been obtained by Prosperetti et al. ${ }^{18}$ Dropping the subscript, the differential equation for $\dot{p}_{g}$ is given, according to Prosperetti, by

$$
\dot{p}=\frac{3}{R}\left(\left.(\gamma-1) K \frac{\partial T}{\partial r}\right|_{R}-\gamma p \dot{R}\right),
$$

where $T$ is the temperature, $K$ is the thermal conductivity of the gas, and $r$ is the radial distance. This equation can be solved simultaneously with the radial equation (1) once the gradient of the temperature field at the bubble wall is found. For this purpose, Prosperetti et al. used the energy equation that can be written as

$$
\frac{\partial \tau}{\partial t}+\frac{(\gamma-1)}{\gamma p R^{2}}\left(\frac{\partial \tau}{\partial y}-\left.\frac{\partial \tau}{\partial y}\right|_{y=1} y\right) \frac{\partial \tau}{\partial y}-D \dot{p}=\frac{D}{R^{2}} \nabla^{2} \tau,
$$

where

$$
\tau=\int_{T_{\infty}}^{T} K(\theta) d \theta
$$

and $T_{\infty}$ is the ambient temperature. For the convenience of having a fixed rather than a moving boundary condition, a new variable was introduced

$$
y=r / R(t) .
$$

The Laplacian operator $\nabla^{2}$ is with respect to the variable $y$, and

$$
D(p, T)=\frac{K(T)}{C_{p} \rho_{l}(p, T)}=\frac{\gamma-1}{\gamma} \frac{K(T) T}{p},
$$

where $\rho$ is the gas density, and $D$ the thermal diffusivity for an ideal gas. The boundary condition for the vector $\tau$ is

$$
\tau(y=1, t)=0 \text {. }
$$

The third formulation used to compare to the experimental results is the Flynn formulation ${ }^{19}$ in which the thermodynamics of the bubble interior are included. Just as in the KM-Prosperetti formulation, Flynn assumes that the pressure inside the bubble is uniform. An improvement from the KM-Prosperetti formulation is that the energy equation in the liquid surrounding the bubble is included. Preliminary tests showed that, for the pressure amplitudes considered here $(\sim 1.5 \mathrm{~atm})$, the temperature in a thin shell of liquid around the bubble is increased significantly during the radial minima. In this respect, Flynn's model should provide additional information on the thermodynamics associated with the bubble motion.

Since the derivation of the Flynn formulation is rather involved, only the radial equation is given here, using Prosperetti's nomenclature, as follows:

$$
\begin{aligned}
\left(1-\frac{\dot{R}}{c}\right) R \ddot{R}+\frac{3}{2} \dot{R}^{2}\left(1-\frac{\dot{R}}{3 c}\right) \\
=\left(1+\frac{\dot{R}}{c}\right) \frac{1}{\rho_{l}}\left[p_{B}(R, t)-p_{A}(t)-P_{\infty}\right] \\
+\frac{R}{\rho_{l} c}\left(1-\frac{\dot{R}}{c}\right) \frac{d p_{B}(R, t)}{d t} .
\end{aligned}
$$

The major difference between this equation and the Keller-Miksis equation is the factor $(1-\dot{R} / c)$ multiplying the time derivative of the pressure just outside the bubble, $d p_{B} / d t$. Comparing the results of the Flynn and KM-Prosperetti formulations, it appears that this extra compressibility term makes a significant contribution when the bubble wall velocities, $\dot{R}$, are high, reducing the strength of the collapse. For the details of the Flynn formulation, the interested reader is referred to Ref. 19.

Five major assumptions were made in deriving these three formulations. Four of them are common to all the models. These are: (a) the bubble remains spherical; (b) the bubble contents obey the ideal gas law; (c) the internal pressure remains uniform throughout the bubble; and (d) no evaporation or condensation occurs inside the bubble. The fifth assumption pertains to the thermodynamic behavior of the liquid surrounding the bubble, and is different in each formulation. These approximations place severe limitations on the models, especially when the pulsations are large and the wall velocities reach values comparable to the speed of sound. For a more detailed consideration of the limitations, see Ref. 24.

The three formulations were solved numerically. The first two, KM-polytropic and KM-Prosperetti, were solved using an IMSL ${ }^{25}$ integration routine. This routine employed Gear's "26 "backward differentiation" technique. A spectral solution scheme developed by Kamath and Prosperetti ${ }^{27}$ was used to solve for the vector $\tau$ in Eq. (6). Flynn's ${ }^{19}$ set of equations was solved using an improved Euler method. Five DEC MicroVaxes in a cluster configuration were used to do the computations.

\section{B. Basic concepts of radial bubble motion}

This section presents some general characteristics of the motion of a single gas bubble driven sinusoidally. Unless otherwise stated, the examples plotted in this section were calculated with the KM-polytropic formulation, which required the least amount of computational time. The other formulations give qualitatively similar results.

In Fig. 1, several examples of bubble response curves, as predicted by the KM-Prosperetti formulation for water at $f=21 \mathrm{kHz}$, have been plotted. Here, the normalized maximum radius $\left(R_{\max } / R_{0}\right)$ during one period of the driving frequency after the solution has reached steady state is plotted as a function of the equilibrium radius $\left(R_{0}\right)$ for several

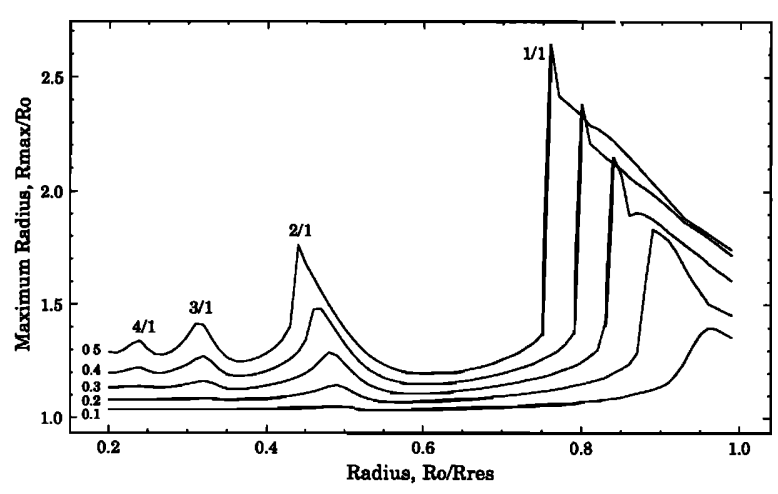

FIG. 1. Theoretical bubble response curves calculated using Prosperetti's formulation for water at $P_{A}=0.1-0.5 \mathrm{~atm}, f=21.0 \mathrm{kHz}$. 
values of the pressure amplitude, $P_{A}$, as indicated by the numbers labeling each curve. Since the steady-state solution has a period equal to that of the driving frequency, $R_{\max }$ is single valued. For $P_{A}=0.1 \mathrm{~atm}(10 \mathrm{kPa})$, the maximum response occurs when the normalized radius $\left(R_{0} / R_{\text {res }}\right)$ is nearly equal to 1 . Other peaks are seen at or near $R_{0} / R_{\text {res }}=1 / 2,1 / 3,1 / 4, \ldots$ etc., each lower in amplitude than the previous one. They are known as the harmonics of the resonance response. These peaks, shown in Fig. 1, have been labeled with an expression $n / m$, known as the order of the resonance according to the notation introduced by Lauterborn. ${ }^{22}$ Here, $n$ and $m$ are defined as follows: If $T_{R}$ is the period of the bubble motion, $T_{f}$ the period of free bubble oscillations, and $T$ the period of the driving frequency, then for a given steady state solution, we can express $T_{R}=m T$ and $T_{R}=n T_{f}$. The case when $m=1$ and $n=2,3$,.. has already been mentioned and are the well-known harmonics, whereas the resonances when $n=1$ and $m=2,3, \ldots$ are called subharmonics. Thus the motion of bubbles near the $m=1$ peaks can be characterized by the number of minima occurring in one acoustic period, $T$, after the solution has reached steady state. For example, bubbles of radii $R_{0} / R_{\text {res }}$ near the $1 / 1$ peak exhibit one radial minimum, bubbles near the $2 / 1$ peak exhibit two radial minima, and so on. Examples of radial pulsations of bubbles near the $1 / 1$ (top) and $2 / 1$ (bottom) peaks are shown in Fig. 2. Since $T_{f}$ is proportional to the bubble radius $R_{0}$, the number of minima occurring in one acoustic period should give some indication of the size of the bubble, with the smaller bubbles having the larger number of minima.

At pressure amplitudes above $1 \mathrm{~atm}(100 \mathrm{kPa})$, the pulsations of small bubbles ( $<40 \mu \mathrm{m}$ ) develop a characteristic profile. An example of a radius-time curve at large pressure amplitudes is shown in Fig. 3 (center). This example is for a $21-\mu \mathrm{m}$ bubble in water at $P_{A}=1.2 \mathrm{~atm}$. In general, the motion follows a relatively slow expansion during the first half of the acoustic cycle when the pressure is negative, followed by a rapid collapse and several rebounds. The word "collapse" here refers to the first bubble contraction after $R_{\max }$ has been reached. The time when the radius reaches a minimum value is usually called the collapse phase, $\phi_{c}$, and is

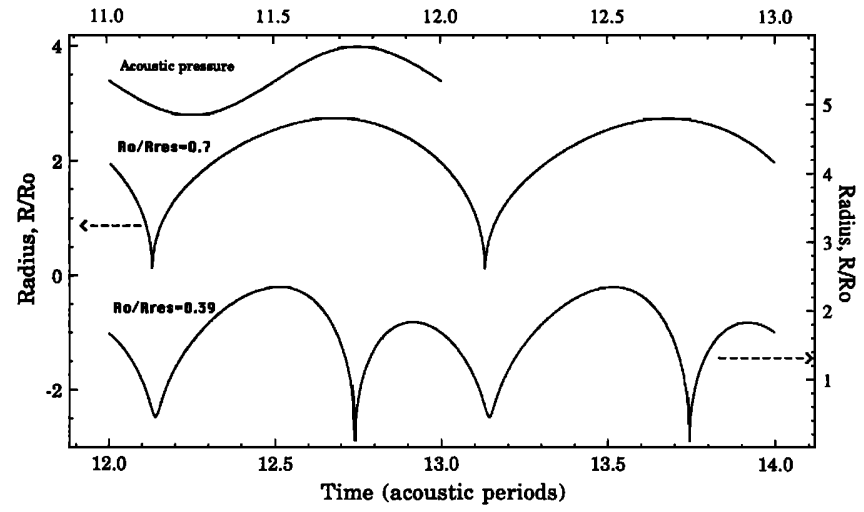

FIG. 2. Theoretical radius-time curves calculated using the polytropic formulation for water at $P_{A}=0.8 \mathrm{~atm}, f=21.0 \mathrm{kHz}, R_{0} / R_{\text {res }}=0.7$ (top), and $R_{0} / R_{\text {res }}=0.39$ (bottom).

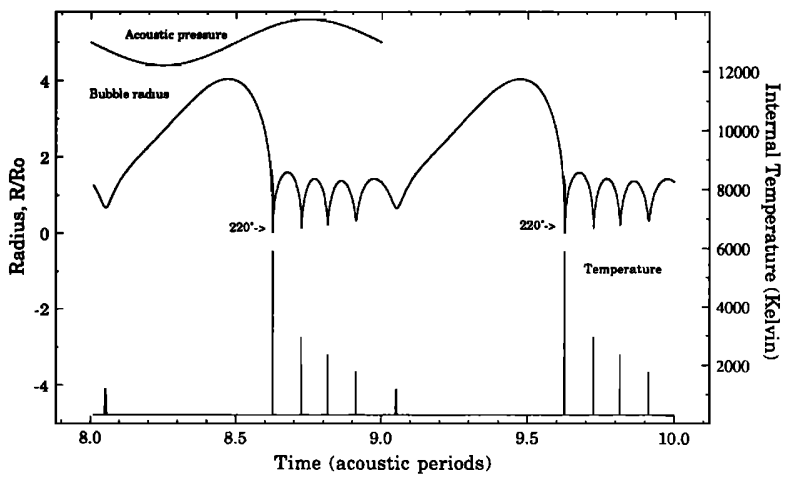

FIG. 3. Theoretical radius- and internal temperature-time curves calculated using Prosperetti's formulation for water at $P_{A}=1.2$ atm, $R_{0} / R_{\text {res }}=0.14, f=21.0 \mathrm{kHz}$.

measured in degrees from the beginning of the negative pressure half-cycle, where $\phi=0^{\circ}$. The beginning of the compression cycle corresponds to $\phi=180^{\circ}$. The collapse in Fig. 3, for example, occurs near $220 \mathrm{deg}$.

The highest temperatures and pressures are generated in the interior of the bubble during this most violent collapse. Figure 3 (bottom) is a plot of the temperature inside the bubble. The large temperature spikes are generated during the violent, nearly adiabatic, collapses. It is during these collapses that sonoluminescence is believed to be generated. Similarly, high pressures are generated in the bubble interior as the gas is rapidly compressed, as shown in Fig. 4 (bottom) for the same conditions as Fig. 3.

Thus three quantities can be used to describe the radial pulsations of bubbles at high pressure amplitudes: the maximum response or pulsation amplitude $\left(R_{\max }\right)$, the phase of the collapse $\left(\phi_{c}\right)$ and the number of minima $(M)$, which includes the first minimum (i.e., the collapse). Note that the number of minima increases as the equilibrium radius decreases.

Up to this point, all quantities have been given a single value for each solution if, after reaching steady state, the solutions have the same period as the driving pressure. But this is not always the case especially at high pressure amplitudes where nonlinear effects are more prominent. In this

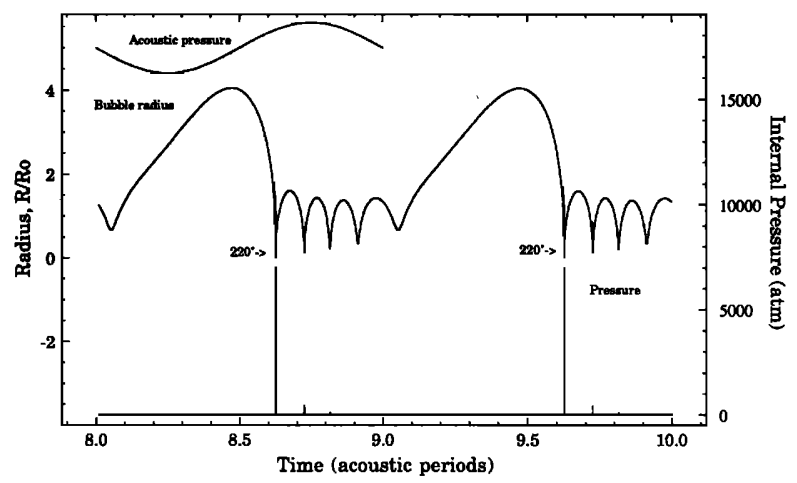

FIG. 4. Theoretical radius- and internal pressure-time curves calculated using Prosperetti's formulation for the same conditions used in Fig. 3. 
regime and under certain conditions, the bubble may display subharmonic motion $(m=2,3, \ldots)$. For example, subharmonic motion of period two $(m=2)$, also known as periodtwo motion, occurs when the period of the radial pulsations is twice the acoustic period $\left(T_{R}=2 T\right)$. Here, the values of $R_{\text {max }}^{i}, \phi_{c}^{i}$, and $M^{i}$, where $i=1,2$, alternate between two different values from one acoustic period to the next. Although period $3,4, \ldots$ etc., motions are theoretically possible, only period-one or period-two solutions were found numerically for the conditions used in the experiments. For the rest of this paper, the superscript $i$ will be dropped from the quantities $R_{\max }, \phi_{c}$, and $M$, unless confusion is likely to arise.

Examples of period-two motion can be found in the response curve shown in Fig. 5 (bottom) calculated for a bubble driven at $1.2 \mathrm{~atm}(120 \mathrm{kPa})$ in water according to the KM-Prosperetti formulation. This type of motion is indicated by a double value of the maximum radius for particular values of $R_{0} / R_{\text {res }}$, and is usually called a period-doubling or pitchfork bifurcation. ${ }^{28}$ The peaks observed in Fig. 5 are due to nonlinear resonances of the radial motion encountered as the equilibrium bubble radius changes; the height of these peaks is usually dependent on the amount of damping included in the theoretical model.

Similarly, a period-two bifurcation is shown in the phase-of-collapse curve at $R_{0} / R_{\text {res }} \approx 0.14(20.3 \mu \mathrm{m})$ in Fig. 5 (top). Not only does the bifurcation occur in the same location, but every change in the curve does as well; i.e., an increase (decrease) in $\boldsymbol{R}_{\max }$ results in an increase (decrease) in $\phi_{c}$. This correlation is not surprising since a larger response requires the bubble to spend more time during the growth and collapse phase. Because a larger pulsation amplitude results in a stronger collapse (other parameters remaining constant), a greater phase of collapse corresponds to a more violent collapse. Thus, after the period-doubling bifurcation occurs, the uppermost branch corresponds to the larger pulsation amplitude and the stronger collapse. A "stronger" collapse means a smaller collapse ratio $\left(R_{\min } / R_{0}\right)$, which generates higher gas densities, temperatures, and pressures. This is illustrated in Fig. 6 where the maximum internal temperature $\left(T_{\max }\right)$ and pressure $\left(P_{\max }\right)$ generated during each period has been plotted on the top and

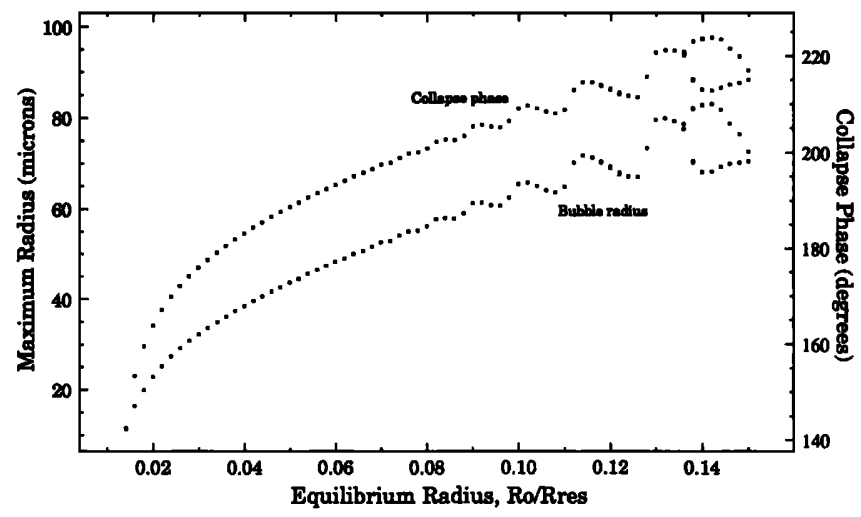

FIG. 5. Theoretical bubble response and phase of collapse curves calculated using Prosperetti's formulation for water at $P_{A}=1.2 \mathrm{~atm}, f=21.0 \mathrm{kHz}$, and $R_{\text {res }}=150 \mu \mathrm{m}$.

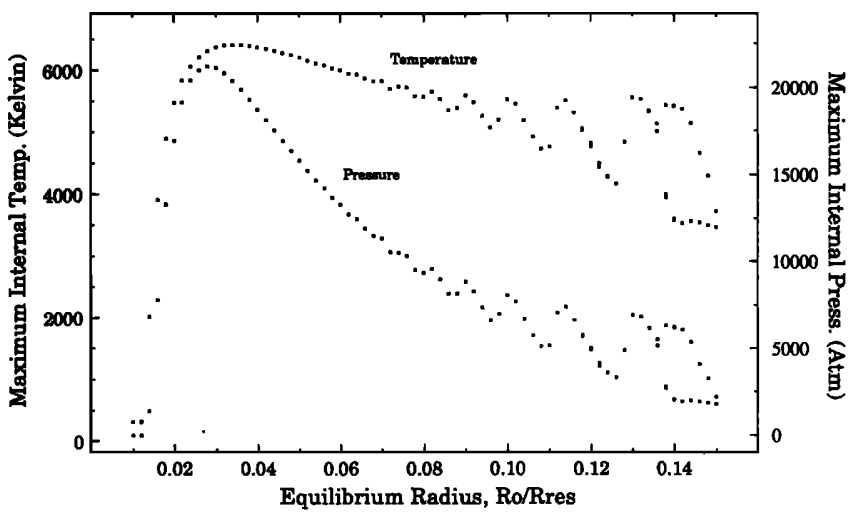

FIG. 6. Theoretical internal temperatures and pressures calculated using Prosperetti's formulation for the same conditions used in Fig. 5.

bottom, respectively, for the same conditions used in Fig. 5 . Here $T_{\max }$ has been averaged over the bubble interior. Again, note that these two quantities are directly correlated to the pulsation amplitude. The close relationship among the quantities $R_{\max }, \phi_{c}, T_{\max }$, and $P_{\max }$ will be useful in the next section where the experimental results will be discussed.

\section{APPARATUS AND EXPERIMENTAL PROCEDURE}

The experiment was divided in two parts: (1) the measurement of the radius-time curve of a single cavitation bubble and (2) the measurement of the phase of the sonoluminescence (SL) emitted by single- and multibubble cavitation fields relative to the phase of the acoustic pressure and to the bubble pulsations.

In both experiments, an acoustic resonator (levitation cell) was used to excite a standing-wave sound field in several glycerine/water mixtures. Using this apparatus, singleand multibubble cavitation was generated at pressure amplitudes in the range $1.0<P_{A}<1.5 \mathrm{~atm}(1 \mathrm{~atm}=100 \mathrm{kPa})$.

In the first part of the experiment, a single bubble was held in position in the levitation cell at amplitudes large enough to generate SL. Light from an Ar-ion laser was scattered from the bubble and detected with a photodiode. The amplitude of the scattered light, modulated by the radial pulsations, was converted to radius via an experimental transfer function in which the average bubble radius was related to the dc component of the scattered intensity. By this method, experimental radius-time $(R-t)$ curves of bubbles pulsating periodically in a steady state were obtained. The radial pulsation amplitude, the timing of the bubble collapse, and the number of rebounds occurring in each acoustic cycle were obtained from the $R-t$ curves.

In the second part of the experiment, SL from a single bubble was detected with a photomultiplier tube in a lighttight enclosure. By using an intermediate reference, namely the pill transducer, the emission of SL was correlated in time with the bubble radius thus obtaining the phase of SL emission relative to the bubble pulsations. The phase of SL relative to the sound field in single- and multibubble cavitation was measured during thousands of consecutive acoustic cycles in order to study the growth, breakup, and steady-state 
motion of bubbles in cavitation fields. The phase was measured with a computer-controlled time-to-amplitude converter.

\section{A. Apparatus}

The basic apparatus used in the experiments consists of a levitation cell plus the driving and controlling electronics. A schematic diagram of this apparatus is shown in Fig. 7(a) (nonshaded blocks). In each experiment, a levitation cell was filled with one of four different water/glycerine mixtures and driven by a 75-W amplifier connected to the cell via an impedance matching coil with an inductance of approximately $4 \mathrm{mH}$. The amplifier was driven by a function generator that was controlled by a process control and data acquisition system.

A levitation cell is a container filled with liquid in which a stationary acoustic wave is excited. The radiation force ${ }^{29}$ exerted on the gas bubble by the stationary wave is used to counteract the hydrostatic or buoyancy force, enabling the bubble to remain suspended in the liquid indefinitely, effectively removed from all boundaries. One cell was made from two 3.6-cm-long $\times 7.5-\mathrm{cm}-0 . d$. concentric piezoelectric cylindrical transducers joined by a glass cylinder of approximately equal dimensions. The transducers were poled to be driven primarily in the thickness mode. A thin, roughly 3mm-thick (i.e., $\ll \lambda_{\text {sound }}$ ) Plexiglas disk was glued to the bottom of the cell, providing a nearly free boundary at the frequencies used in these experiments. A pill-shaped transducer $(0.5 \mathrm{~cm}$ diam $\times 0.3 \mathrm{~cm}$ thick $)$ was attached with epoxy to the outside of the glass to monitor the pressure amplitude and the phase of the acoustic field in the cell.

Besides the cylindrical cell, a rectangular levitation cell was used to photograph and observe the levitated bubbles more clearly. These cells were constructed from one-piece rectangular Pyrex containers with horizontal cross sections of $4.5 \times 4.5,5.1 \times 5.1$, or $6.0 \times 6.0 \mathrm{~cm}^{2}$ and heights of $12.0 \mathrm{~cm}$ with the top end open. A piezoelectric hollow cylindrical
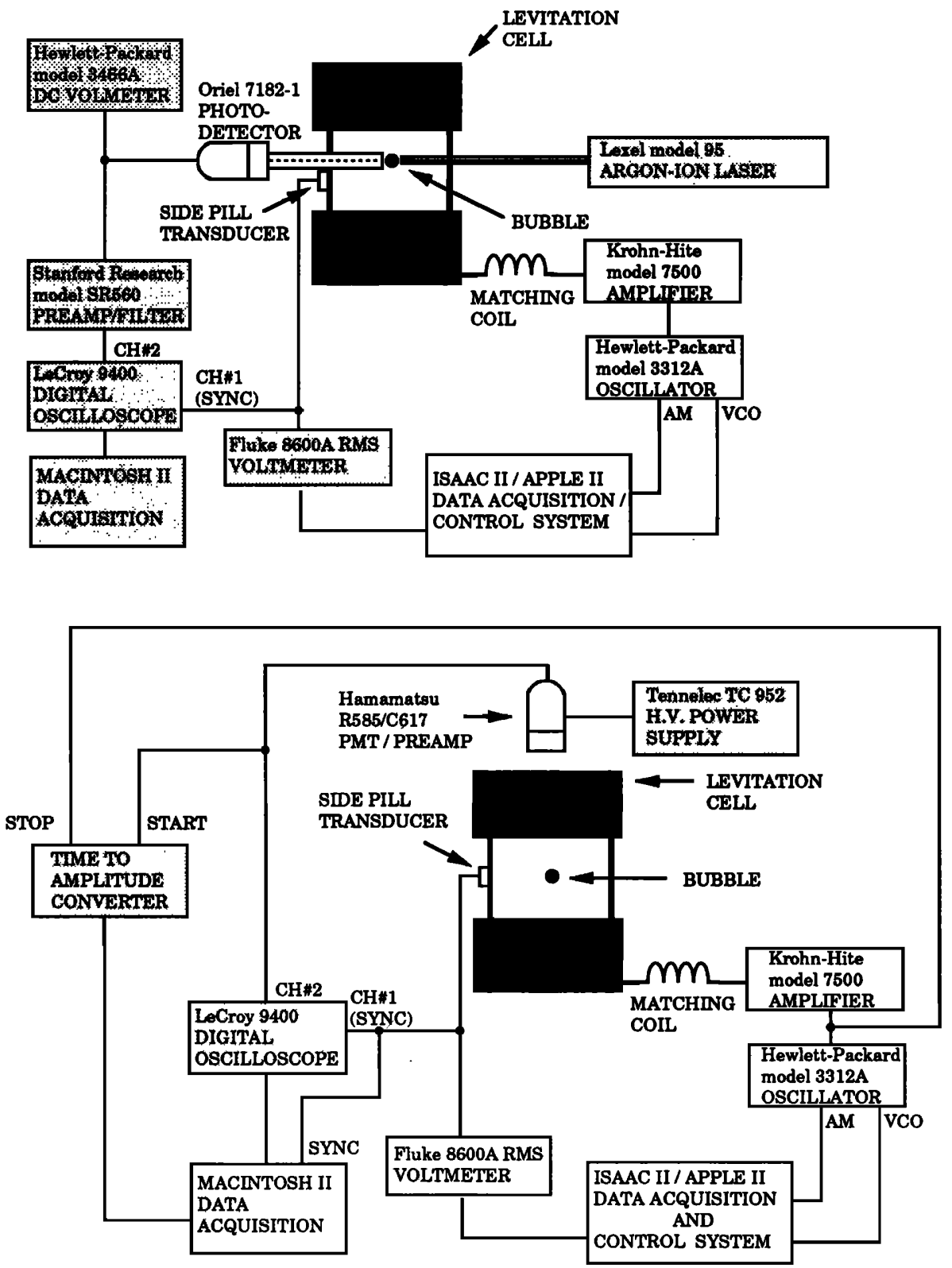

FIG. 7. (a) Schematic diagram of experimental apparatus used to record the scattered light intensity as a function of time. (b) Schematic diagram of experimental apparatus used to measure the phase of sonoluminescence. 
transducer $5 \mathrm{~cm}$ long $\times 5 \mathrm{~cm}$ o.d. was glued to the outside bottom of each container. As with the cylindrical cell, a pill transducer was attached to the outside of a wall and calibrated to provide a noninvasive way to monitor the acoustic pressure in the liquid. Although the rectangular cell was not as stable in frequency and amplitude as the cylindrical one, it provided flat optical surfaces through which gas bubbles could be observed with excellent clarity. Schematic drawings of the levitation cells can be found in Ref. 24.

When driven at its fundamental resonance, a typical levitation cell generated up to 4 atmospheres of acoustic pressure amplitude at the antinodes. During the experiments the cell was always driven at resonance, i.e., between 20 and 25 $\mathrm{kHz}$, depending on the cell and the liquid mixture used. The particular stationary wave excited was determined by the driving frequency. In all the experiments (using a cylindrical cell), an $(r, \theta, z)$ mode of $(1,0,1)$ was used. The cell in use was mounted on a 2 degree of freedom translation stage thus allowing the positioning of the bubble anywhere in the plane perpendicular to the Ar-ion laser. The entire apparatus except for the laser was mounted on an optical table.

To record the radius of the bubble as a function of time, linearly polarized light from a laser was scattered from the bubble using the apparatus shown in Fig. 7(a) (shaded blocks). The scattered light intensity is modulated by the bubble pulsations and, if measured at the appropriate angle ( $\sim 80^{\circ}$ measured from the forward direction), this intensity can be converted to an absolute bubble radius. This technique was developed by Hansen ${ }^{30}$ to size air bubbles under similar conditions and has been used to record small amplitude radial and nonradial bubble oscillations by $\mathrm{Holt}^{31}$ and Horsburgh. ${ }^{32}$ A more detailed description of this technique and of the apparatus can be found in these references.

A 3-W water-cooled Lexel model 95 argon-ion laser operated in the TEM $_{00}$ mode was used as the light source at $0.8-W$ power level and at a wavelength of $488 \mathrm{~nm}$. This power setting was determined empirically by using the maximum power possible before the energy absorbed by the bubble was large enough to affect its motion. An optical rotating polarizer provided a 1200:1 linear polarization ratio. The scattered light was detected by an Oriel 7182-1 photovoltaic silicon photodiode with integral preamplifier, a detection area of $100 \mathrm{~mm}^{2}$ giving a subtended solid angle of $0.04 \mathrm{sr}$. A 488-nm laser line transmission filter was used to reduce background noise. The output of this diode was connected to both a de voltmeter, which provided information on the temporal average radius of the bubble, and via a Stanford Research Systems model SR560 low-noise preamplifier with 40-dB gain, to an ac-coupled LeCroy 9400 digital oscilloscope that recorded the scattered light intensity of the pulsating bubble as a function of time. The data were transferred from the oscilloscope to a Macintosh II computer for analysis and graphical display.

The experimental procedure was straightforward, once the bubble was stabilized in the radial mode. After positioning the bubble in the center of the laser beam, simultaneous traces of the pill transducer output and scattered light intensity were digitized by the oscilloscope and transferred to the computer for permanent storage. The process was repeated for each mixture at several values of the pressure amplitude, $P_{A}$ using the same bubble whenever possible. Each trace corresponded to about $0.2 \mathrm{~ms}$ of real time, i.e., four to five acoustic periods. For the lowest values of the pressure amplitude when the signal-to-noise ratio was too small, time averages over 100 traces were computed on the LeCroy oscilloscope. Because the scattered intensity was periodic over several minutes, no information was lost in the time-averaging procedure.

Figure $7(b)$ is a schematic diagram of the apparatus used to measure the onset and the phase of the light emission relative to the sound field (nonshaded blocks). Because of the low intensity of the light emissions, an 8 - $\mathrm{ft}^{3}$ light-tight box was built that allowed the use of a Hamamatsu model R585/C617 PMT/preamplifier combination designed for single photon counting applications. The dark current and gain specifications of this system were 1 count/s and $10^{5}$, respectively, while the rise and fall times were $15 \mathrm{~ns}$ each. This system provided a time resolution of roughly $1 / 1000$ th of an acoustic period $\left(<1^{\circ}\right)$.

The phase of SL flashes was measured relative to a reference signal consisting of a series of fast electronic pulses ( $\sim 10 \mathrm{~ns}$ wide) generated synchronously with the sound field. By measuring the interval between these pulses and SL events, the phase of SL was determined. A schematic diagram of the circuit used to measure this phase and details of the method can be found in Ref. 24.

The host computer for the data acquisition and control was a MAC II using the Labview ${ }^{\circledR}$ software package. The precision of the data acquisition system was 12 bits, which corresponded to a precision of $\Delta \phi_{c}= \pm 0.1 \mathrm{deg}$ of the phase. The total experimental error, a mixture of electrical, acoustic and optical noise, was estimated at $\pm 1 \mathrm{deg}$.

The general procedure was as follows: After filling the cell with the desired glycerine mixture, an amplitude and phase calibration of the pill transducer was made using the procedure described below. A bubble was then introduced into the liquid and made to luminesce at the desired pressure amplitude. After the light-tight box was closed, the phase of SL was measured for different pressure amplitudes using the TAC system and was stored in the computer for later analysis. The system was periodically verified to be at resonance to maintain the calibration.

Four different glycerine/water mixtures were used in this experiment to obtain data for liquids with several different values of the liquid density, viscosity, surface tension and speed of sound. These mixtures were pure water, GLY21, GLY35, and GLY42, where the two digits represent the percent by weight of glycerine. The solutions were prepared from $99.5 \%$ pure glycerine and deionized water. The mixtures were periodically vacuum-filtered through a $5-\mu \mathrm{m}$ Teflon filter to lower the gas content. This procedure also served to remove impurities after the liquid had been used for some time. The physical parameters of these solutions are shown in Table $\mathrm{I}$.

\section{B. Calibration}

Two different methods were used to determine the absolute acoustic pressure in the levitation cells. One method, the 
TABLE I. Summary of the physical parameters of the solutions used in the experiments.

\begin{tabular}{lcccc}
\hline \hline & $\begin{array}{c}\text { Density } \\
\left(\mathrm{g} / \mathrm{cm}^{3}\right)\end{array}$ & $\begin{array}{c}\text { Viscosity } \\
(\text { centipoise })\end{array}$ & $\begin{array}{c}\text { Surface tension } \\
\left(\mathrm{dyn} / \mathrm{cm}^{2}\right)\end{array}$ & $\begin{array}{c}\text { Speed of sound } \\
(\mathrm{cm} / \mathrm{s})\end{array}$ \\
\hline Water & 1.00 & 1.00 & 71.5 & 148100 \\
GLY21 & 1.05 & 1.54 & 69.4 & 152900 \\
GLY35 & 1.08 & 2.47 & 68.5 & 165700 \\
GLY42 & 1.10 & 3.30 & 68.0 & 169000 \\
\hline \hline
\end{tabular}

levitation technique,,$^{33}$ gave a direct calibration of the pill transducer from which a needle hydrophone (NDL1) was calibrated. A second method was used, the comparison technique, in which the needle hydrophone was calibrated directly using a factory-calibrated reference (B\&K 8103). A single calibration constant for NDL1 was obtained from the average of the two results. The pill transducer was recalibrated at the beginning of each experiment (liquid mixture) using NDL1. During the experiments, the output of the side pill transducer (instead of NDL1) was used to record the acoustic pressure amplitude after being calibrated. The pressure calibration was accurate to within \pm 0.1 atm whereas the precision of the driving electronics was $\pm 0.01 \mathrm{~atm}$.

To determine the instantaneous bubble radius from the scattered light intensity, an experimental intensity-radius transfer curve was needed. Thus bubbles of different sizes were levitated and the scattered light intensity recorded. The size of the bubbles was determined by the rise-time method described in Ref. 24 using the appropriate drag law. The dc component of the measured scattering intensity for bubbles between 20 and $80 \mu \mathrm{m}$ for $42 \%$ glycerine mixture is shown in Fig. 8. The solid line is a second degree polynomial fit to the data. Curves for pure water, $21 \%, 35 \%$, and $60 \%$ glycerine can be found in Ref. 24. The experimental error was estimated to be $\pm 3 \mu \mathrm{m}$.

The phase of the bubble pulsations was measured relative to the output of the calibrated side pill transducer. The phase calibration was made relative to the oscillations of a levitated bubble driven at a low pressure amplitude and far from its resonance frequencies. As illustrated in Fig. 9, the

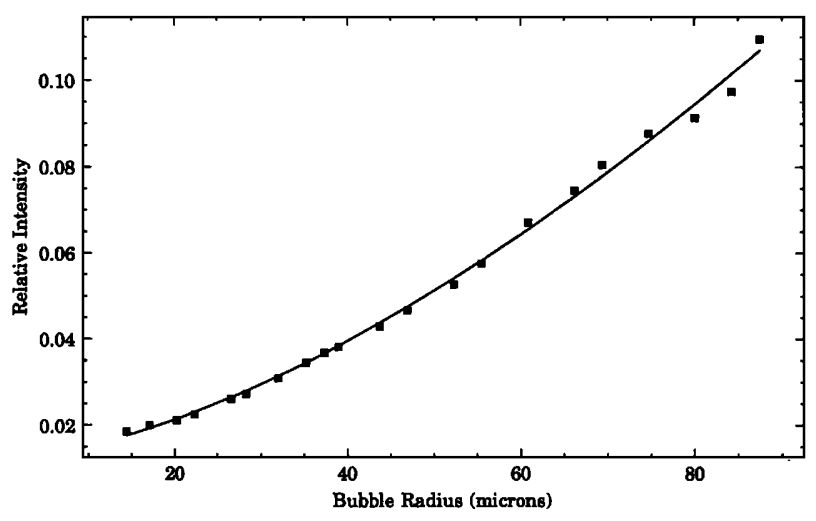

FIG. 8. Measured scattered light intensity versus equilibrium bubble radius for $42 \%$ glycerine. The solid line corresponds to a second degree polynomial fit.

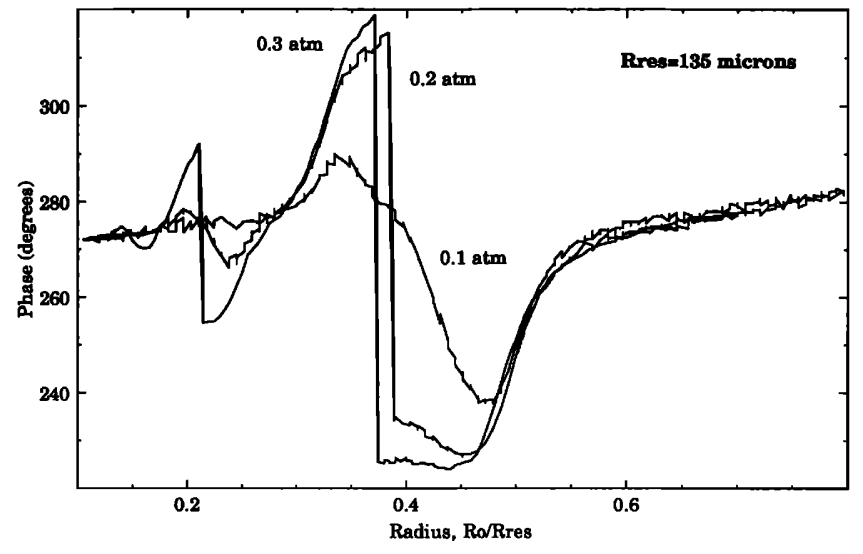

FIG. 9. Theoretical phase of bubble radius minimum of the steady-state solution for $42 \%$ glycerine at $P_{A}=0.1,0.2$, and $0.3 \mathrm{~atm}$ and $f=23.6 \mathrm{kHz}$ used in the phase calibration of the light-scattering apparatus.

theoretical phase of the radial minimum in the region $0.6 R_{\text {res }} \leqslant R_{0} \leqslant 0.75 R_{\text {res }}$ is not only nearly independent of $R_{0}$ but also independent of the pressure amplitude. Since this region corresponds to bubbles away from their harmonic resonances, the phase corresponds to the only radial minimum occurring each cycle of the driving pressure. This phase was also found to be independent of the mathematical formulation used. Thus, by knowing the phase of the bubble pulsations relative to the sound field, the phase of the pill transducer also could be found. Bubbles in this size range were levitated and the simultaneous oscilloscope traces of both the pill transducer output and scattered light intensity were stored in the computer. The two traces were displayed on the Macintosh II using graphics software. The time difference between minima was measured and the phase difference was calculated. The total error of the calibration procedure was estimated to be $\pm 5 \mathrm{deg}$. This error was mostly due to the uncertainty in the measurement of the bubble size.

\section{RESULTS AND DISCUSSION}

\section{A. General observations}

The initial goal of this study was to find the range of SLactive bubble radii in a cavitation field at pressure amplitudes above 1 atm $(100 \mathrm{kPa})$, that is, above the pressure threshold for detectable light emission. The goal was achieved by measuring the phase of SL relative to the sound field and comparing it to the phase of bubble collapse predicted by the different theories of radial bubble pulsations. Since the SL flashes are very short, if they are emitted at the time of the bubble collapse (shown later), one can determine the phase of collapse by measuring the phase of SL. According to the theories, the phase of bubble collapse varies over a wide range of values depending on the pressure amplitude and the bubble size, as shown in Fig. 5. It is thus possible to estimate the bubble sizes that are active during cavitation by measuring the phase of SL emission, assuming the theories are correct and that SL is emitted during the collapse of the bubble.

During the initial experiment, it was found that the scatter in the phase of SL at times decreased until it was less than 
\pm 1 deg-i.e., below the noise level-indicating that the phase was constant in time. It was observed that as the pressure was increased the degassing action of the sound field was reducing the number of bubbles, causing the cavitation streamers to become very thin until only a single bubble remained. The remaining bubble was approximately $20 \mu \mathrm{m}$ in radius and positioned at the antinode. At this point, the bubble was remarkably stable in position and shape, remained constant in size, and seemed to be pulsating in a purely radial mode. With the room lights dimmed, a greenish luminous spot the size of a pinpoint could be seen with the unaided eye, near the bubble's position in the liquid. The luminous spot was then located at the bubble's geometric center when observed through a microscope. Although this luminescence had been observed before by other researchers, ${ }^{1}$ very sensitive instruments were usually required. Furthermore, it had never been observed for a single, stable bubble.

A single, radially pulsating bubble was stabilized in slightly degassed water-glycerine mixtures with glycerine concentrations less than $60 \%$. A description of the visual observations of the stabilization process as the acoustic pressure was increased follows: After injecting a gas bubble with a small syringe at about $P_{A}=0.6 \mathrm{~atm}(60 \mathrm{kPa})$, the bubble exhibited dancing motion indicative of surface waves and asymmetric collapses. ${ }^{34}$ As the pressure amplitude was increased, the dancing motion became more vigorous, causing the bubble to fragment. A bubble cluster was then formed as the residual bubbles moved around the parent bubble. The bubble system looked more like a cloud and very often developed into what has been termed a "shuttlecock." When observed through a microscope, the shuttlecock appeared to be a cloud of microbubbles surrounding a larger bubble approximately $50 \mu \mathrm{m}$ in radius. Through the interaction with the sound field, this cloud developed a definite pattern of motion from one side of the bubble to the other. In contrast with single bubbles, the position of the shuttlecock was not on the levitation cell's axis but a small distance away from it (0.5$1.0 \mathrm{~cm}$ ). Microbubbles appeared to be ejected from the side of the cloud directly away from the antinode and were immediately attracted toward the cloud by Bjerknes forces, ${ }^{35}$ so that they moved around the cloud toward the antinode. As the microbubbles reached the opposite end of the bubble cloud from which they had been ejected, they were pulled in, possibly by interbubble forces directed toward the center of the cloud. Thus, a rotational pattern was established. The shuttlecock was observed to emit a low rate of SL flashes.

It is important to note that a well-defined pressure threshold, $p_{t}^{\mathrm{sT}}$, was observed at which stability was reached. In addition, a hysteresis effect was observed, i.e., after reaching stability, the pressure could be decreased below the threshold $p_{\mathrm{t}}^{\mathrm{ST}}$ and stability was maintained. In general, stable bubbles could be driven at values of the acoustic pressure 0.1 atm below the stability threshold. Furthermore, if driven above a certain pressure the bubble disappeared, possibly due to dynamic instabilities. For convenience, we will call these the lower, $p_{\mathrm{lt}}^{\mathrm{ST}}$, and upper, $p_{\mathrm{ut}}^{\mathrm{ST}}$, stability thresholds, respectively, where the subscripts indicate lower and upper threshold and the superscript indicates a stability (rather than instability) threshold. These thresholds are illustrated in Fig. 10. In water, for example, $p_{\mathrm{t}}^{\mathrm{ST}}$ was measured to be 1.2 atm, $p_{\mathrm{lt}}^{\mathrm{ST}}$ at $1.1 \mathrm{~atm}$, and $p_{\mathrm{ut}}^{\mathrm{ST}}$ at $1.3 \mathrm{~atm}$. The range of pressures at which bubbles were stable was $1.1 \leqslant P_{A} \leqslant 1.5 \mathrm{~atm}$ depending on the liquid mixture. For water, it was $1.1 \leqslant P_{A} \leqslant 1.3 \mathrm{~atm}$ whereas for a $42 \%$-glycerine solution it was $1.3 \leqslant P_{A} \leqslant 1.5$ atm. Thus higher concentrations of glycerine required slightly higher pressures to achieve radial stability. It was also found that these ranges could be enlarged (toward the lower pressures) by decreasing the dissolved gas content of the liquid. Also, as the amount of dissolved gas increased, regions of instability within the stability pressure range began to appear; however, the upper pressure threshold, $p_{\mathrm{ut}}^{\mathrm{ST}}$, at which the bubble disappeared, remained constant. It was also concluded that rectified diffusion ${ }^{36}$ played an important role in the stability process.

The observation of an upper pressure amplitude threshold $p_{\mathrm{ut}}^{\mathrm{sT}}$, above which pulsating bubbles cannot persist, seems to confirm the existence of a transient cavitation threshold as defined by Flynn. ${ }^{7,8}$ According to Flynn, ${ }^{8}$ the motion of bubbles pulsating with an expansion ratio $\left(R_{\max } / R_{0}\right)$ above a certain value become inertia controlled. The collapse of these bubbles tends to be violent, often resulting in the destruction of the bubbles. The bubbles studied here $(\sim 20$ $\mu \mathrm{m}$ ) fall under the "large" category ( $>5 \mu \mathrm{m}$ ) which, according to the Flynn model, should exhibit thermally related effects (e.g., sonoluminescence) before reaching the transient cavitation (dynamical) threshold. For $20-\mu \mathrm{m}$ bubbles, Flynn predicted a dynamical threshold of $R_{\max } / R_{0} \approx 2.2$. These predictions appear to be confirmed by the observations made here, since SL has been observed from stably

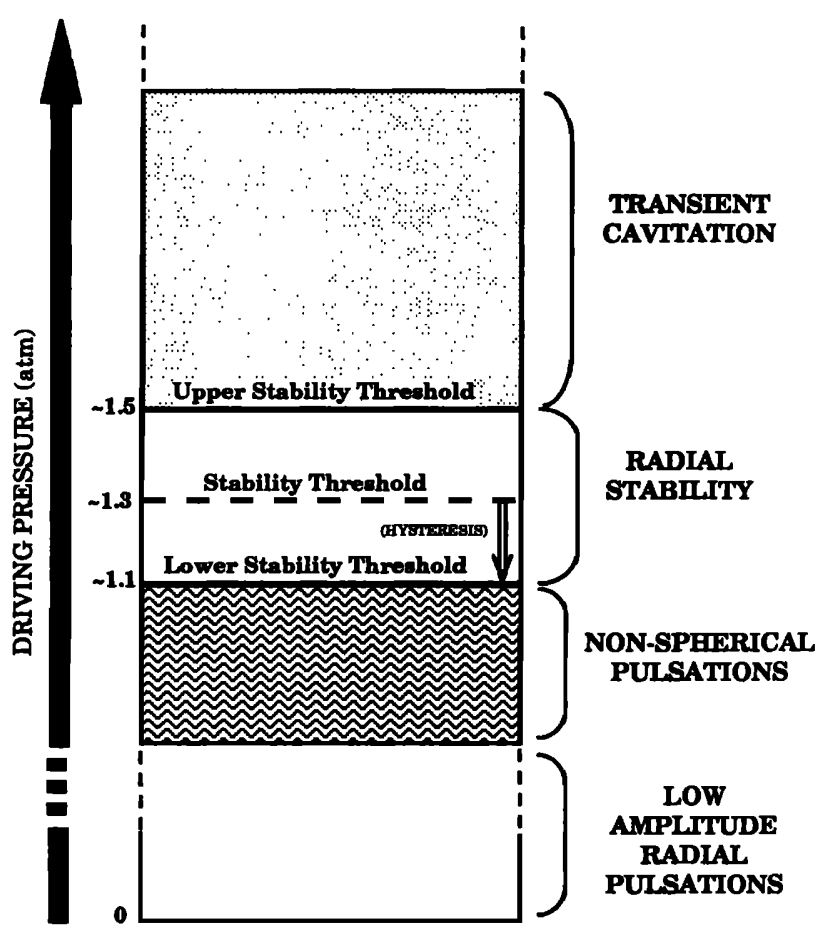

FIG. 10. Diagram of the observed radial stability thresholds for 15- to 20$\mathrm{mm}$ bubbles in water/glycerine mixtures in an acoustic levitation system at $f=21-25 \mathrm{kHz}$. 
pulsating bubbles. After reaching a value of the expansion ratio $R_{\max } / R_{0} \approx 4$, the bubbles become unstable, i.e., they disintegrated. Although the observed values of the dynamical threshold differ somewhat from those predicted by Flynn, it is generally agreed that these theoretical values "most likely act as lower limits to experimental thresholds." 8

Although the observed upper threshold instability can be explained in terms of Flynn's model, the region of stability above the previously measured surface wave threshold is a totally new and unexpected observation for which an adequate explanation in terms of the equations of single radial bubble pulsations has not been found. Despite the lack of understanding about the particular mechanisms involved, the discovery of a single bubble pulsating at large amplitudes has many important consequences. Among these is the ability to obtain new and more accurate data about the motion of bubbles in cavitation fields, such as the radius-time curve of single bubbles pulsating radially from which we can measure $R_{\max }, \phi_{c}$, and $M$ to test the applicability of the theories. One objective of this study, therefore, is to determine which value or values of $R_{0}$ give the best agreement between a particular theory and experiment for these three independently measured parameters.

Comparison between the measurements and KM-Prosperetti model will be made first followed by a comparison with the other models with the intent of determining the most accurate theory. The theoretical values of the internal temperatures, pressures and relative densities predicted by the KM-Prosperetti and Flynn formulations will be compared. The theoretical temperature calculated at the experimentally determined threshold for SL will be discussed and compared with previous measurements of temperature. In addition, the simultaneity of SL and the collapse of the bubble will be verified. We will present the results of the single bubble experiments first, along with the theoretical results. Finally, the observations in multibubble cavitation fields will be discussed.

\section{B. Light scattering experiments}

\section{Experimental data versus the KM-Prosperetti theory}

The light scattering apparatus described in Sec. II was used to obtain the radius versus time curves for radial pulsations of bubbles. The value of the equilibrium radius of the bubble $\left(R_{0}\right)$ was obtained by the rise-time method, ${ }^{24}$ although thermal and acoustic pressure currents in the liquid sometimes made this measurement both difficult and imprecise. From these measurements, it was determined that the equilibrium bubble sizes were roughly $20 \pm 5 \mu \mathrm{m}$ for all the liquid mixtures.

Figure 11 shows a plot of the bubble radius versus time (dotted line) at $P_{A}=1.22 \mathrm{~atm}$ in GLY21 as measured by the photodetector. This figure is a single trace obtained from the LeCroy oscilloscope. The $y$ values have been converted from intensity to radius using the transfer function obtained during the calibration procedure. This figure is a typical example of a radius-time $(R-t)$ curve obtained from a single bubble pulsating at large amplitudes with a period equal to that of the driving pressure. Here, $R-t$ curves for a range of

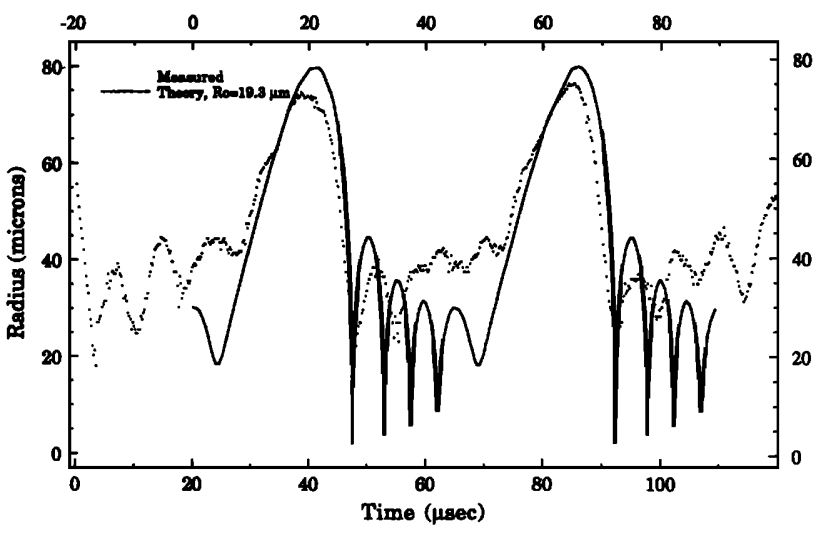

FIG. 11. Theoretical (solid) and experimental (dotted) radius-time curve obtained with the light-scattering apparatus in $21 \%$ glycerine at $P_{A}=1.22$ atm and $f=22.3 \mathrm{kHz}$.

pressures between 1.1 and $1.5 \mathrm{~atm}$ were obtained for all the liquid mixtures. All the $R-t$ curves obtained at these high driving pressure amplitudes are characterized by a relatively slow expansion ( $\sim 15-20 \mu \mathrm{s})$, followed by a rapid collapse ( 5-10 $\mu$ s) after which several rebounds occur before the next cycle starts. Curves similar to Fig. 11 were also obtained for the other mixtures and representative curves can be found in the Appendix of Ref. 24. The number of radial minima during one cycle was usually 4 or 5 , although three minima occurred occasionally. The differences in the traces taken in different liquids were mainly in the amplitude of the pulsations and the phase of the first radial minimum.

For comparison, a theoretical $R-t$ curve using the same experimental conditions with $R_{0}=19.3 \mu \mathrm{m}$ has been plotted in Fig. 11 (solid line). The reasons for choosing this value of $R_{0}$ will be explained below. The general shape of the two plots is very similar, including the magnitude of the bubble response and the existence of rebounds after the collapse. Discrepancies were found in the number of minima and the apparent increase in the amplitude of the rebounds in the experimental $\boldsymbol{R}-\boldsymbol{t}$ curve (dotted line), as opposed to the decrease predicted by the theory (solid line). Some of these discrepancies will be discussed below. Periodic pulsations such as those seen in Fig. 11 were observed continuously for thousands of acoustic periods. These $R-t$ curves and the observation of the spherical outline of the bubble pulsations through the microscope when bubbles were levitated in the rectangular cell are most convincing evidence of the periodicity, stability, and spherical symmetry of the bubble pulsations.

Several aspects of these nonlinear bubble pulsations have been measured using the $R-t$ curves of single bubbles pulsating at large amplitudes $\left(R_{\max } / R_{0}>3\right)$. In the following sections, some of these measurements will be presented and compared to the theoretical values predicted by the different formulations. Since it was not possible to measure the equilibrium bubble size precisely, calculations were made for $R_{0}=15,20$, and $25 \mu \mathrm{m}$. Although the mechanism by which the bubbles are stabilized is not well understood, each bubble did appear to remain constant in size for a given set of acoustical parameters (pressure amplitude, gas concentration, etc.). 
a. Pulsation amplitude. For each $R$ - $t$ curve obtained, the pulsation amplitude $R_{\max }$ was measured and plotted versus $P_{A}$ for water and GLY42 as shown in Figs. 12 and 13; data for GLY21, GLY35, and GLY60 can be found in Ref. 24. In the same figures, values of the theoretical predictions made by the KM-Prosperetti model for the same experimental conditions have been plotted for $R_{0}=15,20$, and $25 \mu \mathrm{m}$, as indicated in the legend. Results for the KM-polytropic and Flynn theories for 15 and $20 \mu \mathrm{m}$ have also been included and will be discussed later. The data shown in Figs. 12 and 13 are mean values taken from stored oscilloscope traces. The traces were taken using the same bubble and each trace consisted of several acoustic periods for each value of $\boldsymbol{P}_{A}$. Usually, only one trace was stored for each value of $P_{A}$ due to the large amount of time and storage space that each trace required. The error bars placed on these data represent the combined experimental uncertainty estimated to be \pm 5 $\mu \mathrm{m}$. Some theoretical curves exhibit abrupt changes caused by resonances of the radial motion excited at different values of $P_{A}$. Bifurcations into period-2 and period-4 solutions also occur, especially for the 20 - and $25-\mu \mathrm{m}$ bubbles. These resonances and bifurcations have been briefly discussed in Sec. I. Although values were calculated in increments of $P_{A}=0.01 \mathrm{~atm}$ for each value of $R_{0}$, not all the data were displayed. These gaps in the theoretical curves indicate that no steady-state solutions were found in a reasonable amount of time, usually 20 periods of the driving pressure.

In general, comparison of the pulsation amplitude data indicates better agreement with an equilibrium radius of 15 $\mu \mathrm{m}$, although this is not always true. Some experimental data, for example, agrees partially with the theoretical results of a $20-\mu \mathrm{m}$ bubble. It should be noted here that it was probable that the equilibrium bubble radius changed as the pressure amplitude was increased. In fact, for GLY21, closer agreement with the theoretical results was found using the $15-\mu \mathrm{m}$ bubble at the lower pressures, but with the $20-\mu \mathrm{m}$ bubble at the higher pressures. This effective increase in equilibrium radius with pressure amplitude may have resulted from rectified diffusion ${ }^{36}$ which, in general, is proportion-

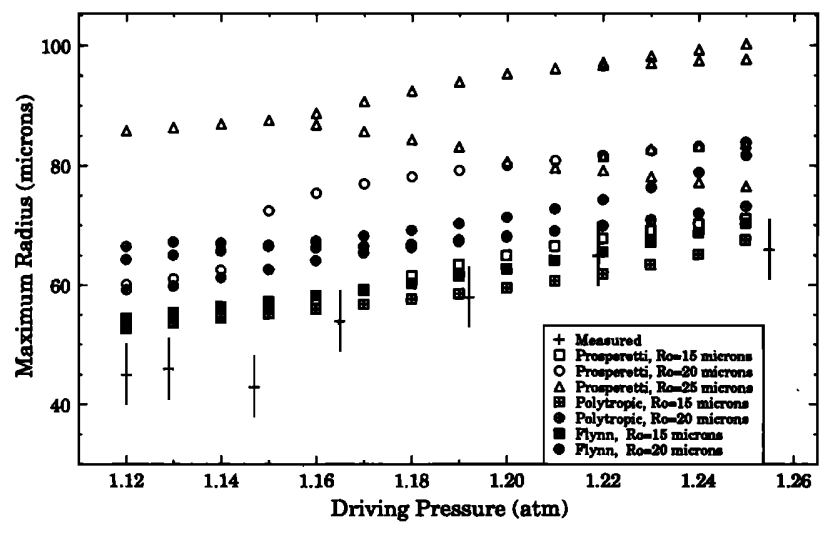

FIG. 12. Measured and theoretical pulsation amplitude $\left(\boldsymbol{R}_{\max }\right)$ versus acoustic pressure amplitude in water using all three formulations at $f=21.0$ kHz.

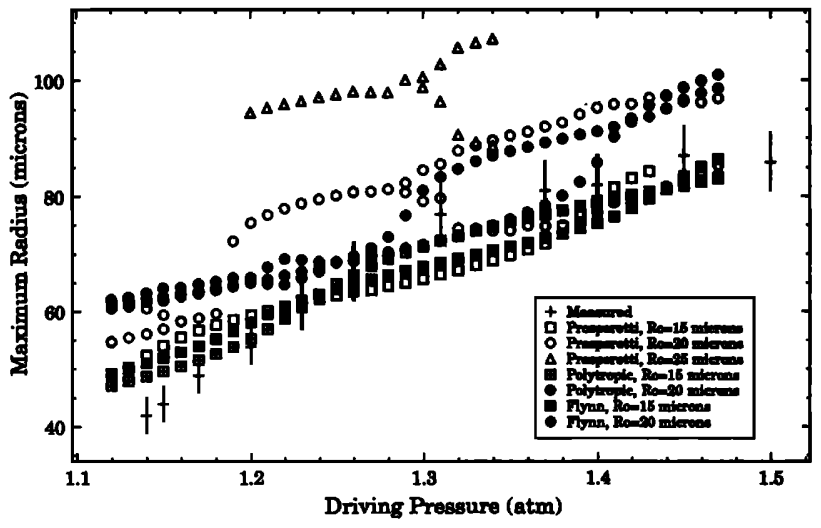

FIG. 13. Measured and theoretical pulsation amplitude $\left(\boldsymbol{R}_{\max }\right)$ versus acoustic pressure amplitude in GLY42 using all three formulations at $f=23.6 \mathrm{kHz}$.

al to $P_{A}$. On the other hand, these data may reflect a change in the applicability of the theories as $P_{A}$ is increased.

Theoretically, resonances and bifurcations occur in different regions of the bubble response curve, the exact locations being very sensitive to the specific set of parameters values, even for the same values of $P_{A}$ and $R_{0}$. Thus it was not expected to find agreement between theory and experiment in the regions where rapid transitions occurred. It is noted that although subharmonic motion was predicted often for the range of parameters considered, it was never observed in these experiments.

$b$. Phase of collapse. From the same $R-t$ curves considered in the previous section, the phase of collapse $\phi_{c}$ was measured. The results are plotted in Figs. 14 and 15 with the theoretical predictions of the KM-Prosperetti formulation for 15-, 20-, and 25- $\mu \mathrm{m}$ bubbles and the KM-polytropic and Flynn theories for $15-$ and $20-\mu \mathrm{m}$ bubbles. The estimated total error of the calibration procedure was $\pm 5 \mathrm{deg}$ and it is indicated by the error bars.

The results of the phase of collapse measurements were very similar to the pulsation amplitude measurements in the

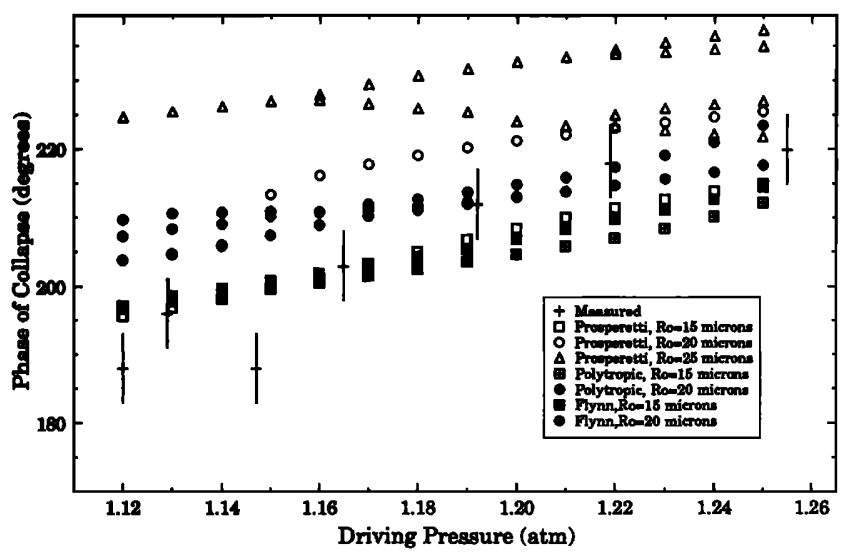

FIG. 14. Measured and theoretical phase of bubble collapse $\left(\Phi_{c}\right)$ versus acoustic pressure amplitude in water using all three formulations at $f=21.0$ kHz. 


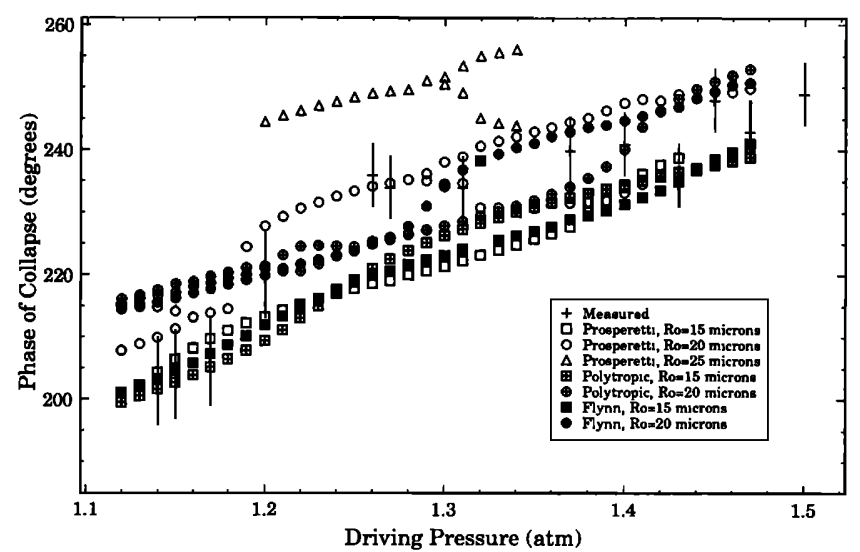

FIG. 15. Measured and theoretical phase of bubble collapse $\left(\Phi_{c}\right)$ versus acoustic pressure amplitude in GLY42 using all three formulations at $f=23.6 \mathrm{kHz}$.

previous section. In summary, these results can be interpreted as follows: In Fig. 14 (water), $R_{0}$ appears to increase from $10 \leqslant R_{0} \leqslant 15 \mu \mathrm{m}$ to $15 \leqslant R_{0} \leqslant 20 \mu \mathrm{m}$ as $P_{A}$ increases, whereas in Fig. 15 (GLY42), $R_{0} \approx 15 \mu \mathrm{m}$ at low $P_{A}$ and $R_{0} \approx 20 \mu \mathrm{m}$ at high $P_{A}$. The collapse phase data for water predicted larger radii than the pulsation amplitude data shown in the previous section, although the discrepancy was small enough to be within the experimental error.

c. Number of radial minima. A third experimental determination that can be used to test the theory is the number of radial minima observed during one acoustic period. This parameter is mainly dependent on the bubble size, although some dependence on the pressure amplitude was found from the calculations. Figures 16 and 17 show the number of minima measured in each liquid mixture and those predicted by the KM-Prosperetti and the KM-polytropic models for 15, 20 , and $25 \mu \mathrm{m}$ and the Flynn theory for $15-$ and $20-\mu \mathrm{m}$ bubbles. Noninteger values of the measured number of minima indicate that two different values were observed in the same radius-time curve; In this instance, the mean value was plotted. The error bars indicate the estimated error in the measurement. Double values in the theoretical data mean that a different number of minima occurred per period in the steady-state solution, usually due to subharmonic motioni.e., $m>1$ (see Sec. I).

The number of minima observed were, in general, fewer than the number expected from the theoretical predictions based on the pulsation amplitude and collapse phase measurements. This means that the equilibrium bubble radii predicted based on the number of minima were larger since, as shown previously, larger bubbles pulsate with fewer minima. For instance, Fig. 16 (water) predicts $R_{0} \approx 20 \mu \mathrm{m}$ at low values of $P_{A}$ and $R_{0} \approx 25 \mu \mathrm{m}$ at the higher values of $P_{A}$. Figure 17 (GLY42) predicts $R_{0} \approx 20 \mu \mathrm{m}$ remaining constant for the range of $P_{A}$ considered. It is not clear why the number of minima observed was fewer than predicted by the theories. The explanation may be linked to another anomalous observation in the rebounds of the radius-time curves. As shown in Fig. 11, the bubble rebounds appear to increase

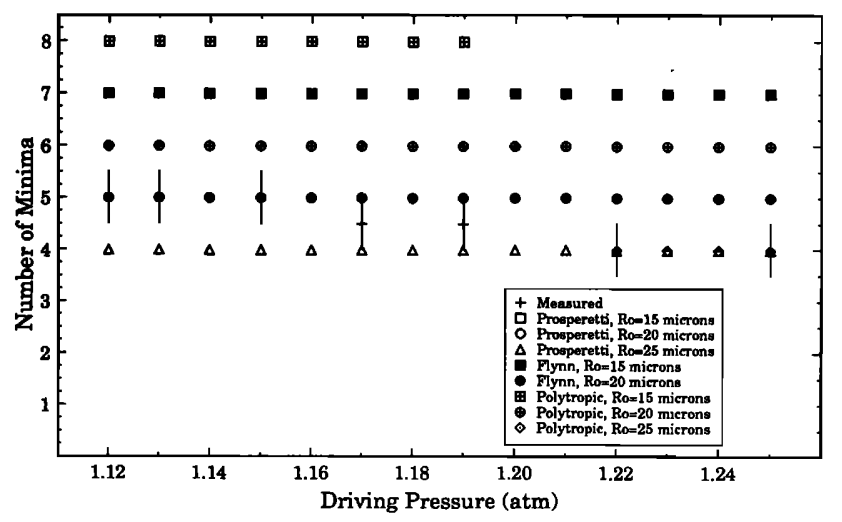

FIG. 16. Measured and theoretical number of minima $(M)$ versus acoustic pressure amplitude in water using all three formulations at $f=21.0 \mathrm{kHz}$.

in radius within each period instead of decreasing as predicted by the theoretical $R-t$ curve. The experimental $R-t$ curves also seem to indicate much larger amplitudes for the rebounds. Larger amplitudes would indeed result in fewer rebounds because each rebound would require more time. In this respect, the observed fewer number of minima is consistent with the large rebound amplitudes observed in the scattered-light data. No explanation has been found for this increase in the amplitude of the rebounds.

Additional evidence on the size of the bubbles can be obtained by considering the periodicity of the solutions. For example, period-two solutions were often predicted by the theory for the $20-\mu \mathrm{m}$ bubble. For the $25-\mu \mathrm{m}$ bubble, few, stable, period-one solutions were obtained whereas all the solutions for $R_{0}=15 \mu \mathrm{m}$ were stable and with the same period as that of the driving pressure. In this respect, the fact that only bubble pulsations with the same period as that of the sound field were observed in the laboratory suggests that the bubble radius was less than $20 \mu \mathrm{m}$.

In summary, the data presented in the last three sections may be interpreted as indicating that the equilibrium radii of

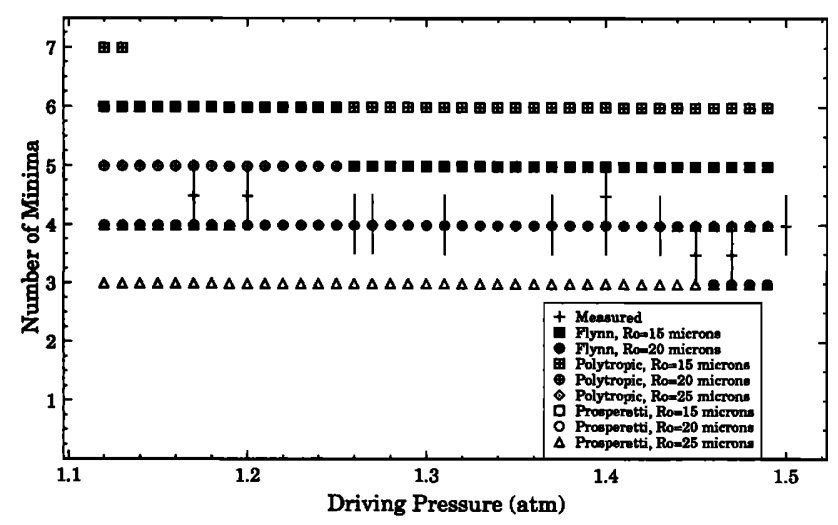

FIG. 17. Measured and theoretical number of minima $(M)$ versus acoustic pressure amplitude in GLY42 using all three formulations at $f=23.6 \mathrm{kHz}$. 
stabilized bubbles driven at pressure amplitudes between 1.1 and $1.5 \mathrm{~atm}$ in different water/glycerine mixtures were between 15 and $20 \mu \mathrm{m}$. It may also be inferred from some of the data that the equilibrium bubble radii increased as the driving pressure was increased. The predicted values of $R_{0}$ based on the measurements of $R_{\max }$ and $\phi_{c}$ are consistent for three of the five data sets (see Ref. 24). Inconsistencies were found in the predictions for water, GLY35, and GLY42. The measured number of minima indicated, on the other hand, that the equilibrium bubble radii were larger-between 20 and 25 $\mu \mathrm{m}$. This lack of total agreement means that the KM-Prosperetti theory, although applicable, may not give a quantitatively accurate description of a cavitation bubble pulsating at high amplitudes.

\section{The KM-polytropic and Flynn theories}

Let us first consider the results from the Flynn model. For clarity, only values of $R_{0}=15$ and $20 \mu \mathrm{m}$ have been plotted using the solid symbols as shown in the legend. Extrapolated values were estimated for the Flynn and the KMpolytropic model data when necessary. The pulsation amplitude data shown in Fig. 12 (water) indicate that $R_{0} \leqslant 15 \mu \mathrm{m}$, increasing as $P_{A}$ increases. The data shown in Fig. 13 (GLY42) indicate that $R_{0} \leqslant 15 \mu \mathrm{m}$, with $R_{0}$ increasing to 15 $\mu \mathrm{m}$ as $\boldsymbol{P}_{A}$ was increased. However, the phase of the collapse data shown in Fig. 14 (water) suggests that $10 \leqslant R_{0} \leqslant 20 \mu \mathrm{m}$ with $R_{0}$ also increasing as $P_{A}$ increases. The data in Fig. 15 (GLY42) indicate that $15 \leqslant R_{0} \leqslant 20 \mu \mathrm{m}$, with $R_{0}$ increasing with $P_{A}$. The number of minima data shown in Fig. 16 (water) imply that $20 \leqslant R_{0} \leqslant 25 \mu \mathrm{m}$, with $R_{0}$ increasing with $P_{A}$ and those in Fig. 17 (GLY42) indicate that $R_{0} \approx 20 \mu \mathrm{m}$ also increasing slightly with $P_{A}$. Note that, in general, the conclusions agree well with KM-Prosperetti in the previous section.

Let us now consider the results from the KM-polytropic model plotted in the same figures using the crossed symbols as shown in the legend. The pulsation amplitude data shown in Fig. 12 (water) indicate that $10 \leqslant R_{0} \leqslant 20 \mu \mathrm{m}$, with $R_{0}$ increasing as $P_{A}$ increases. The data shown in Fig. 13 (GLY42) indicate that $R_{0} \leqslant 15 \mu \mathrm{m}$, with $R_{0}$ increasing to 15 $\mu \mathrm{m}$ or more as $P_{A}$ increases. The phase of collapse data shown in Fig. 14 (water) suggest that $10 \leqslant R_{0} \leqslant 20 \mu \mathrm{m}$ with $R_{0}$ also increasing as $P_{A}$ increases. The data in Fig. 15 (GLY42) indicate that $15 \leqslant R_{0} \leqslant 20 \mu \mathrm{m}$, with $R_{0}$ increasing with $P_{A}$. The number of minima data shown in Fig. 16 (water) imply that $22 \leqslant R_{0} \leqslant 25 \mu \mathrm{m}$, with $R_{0}$ increasing with $P_{A}$. The data in Fig. 17 (GLY42) indicate that $R_{0} \approx 20 \mu \mathrm{m}$, with $R_{0}$ also increasing slightly with $P_{A}$. In general, these values of $R_{0}$ agree well with those based on the KM-Prosperetti predictions shown in the previous sections, and even better with those based on the Flynn predictions.

In summary, two conclusions can be made: (1) The measurements do not constitute a good test to discriminate between the theories, since the experimental error is larger than the difference between the values predicted by the theories using the same conditions; (2) each of the theories provide excellent qualitative and reasonable quantitative descriptions of the dynamic behavior of stable cavitation bubbles driven in a highly nonlinear regime.

\section{Temperature, pressure, and relative density}

The maximum internal temperatures, pressures and relative densities have been summarized in Tables II, III, and IV, respectively. Because of the isothermal bubble motion of the KM-polytropic formulation, the predicted pressures were unreasonably high and the temperatures unreasonably low. We have, therefore, not included them in this analysis. In order to make a meaningful comparison between KMProsperetti and Flynn formulations the maximum temperatures calculated from KM-Prosperetti have been spatially averaged over the bubble radius. The relative densities, which have been normalized with respect to equilibrium conditions, were calculated from $\rho_{\max } / \rho=\left(R_{0} / R_{\min }\right)^{3}$, i.e., assuming no condensation or vaporization occurs. Note that for water, $1.12 \leqslant P_{A} \leqslant 1.25$ whereas for GLY42, $1.12 \leqslant P_{A} \leqslant 1.47$ so that higher values are expected for GLY42.

The third column of each table contains the maximum internal temperatures $\left(T_{\max }\right)$, pressures $\left(P_{\max }\right)$, and relative densities $\left(\rho_{\max }\right)$ predicted by the KM-Prosperetti theory, and those predicted by the Flynn theory are shown in the fourth column. Note that, in every case, the temperatures and pressures predicted by the KM-Prosperetti theory are higher than those predicted by Flynn for the same bubble size, even for the cases where the pulsation amplitude predicted by KM-Prosperetti was less. The fifth column of each table contains the same quantities calculated using the Prosperetti formulation with Flynn's radial equation (instead of Keller-Miksis). These values will be referred to as F-Prosperetti. The difference in the predictions shown in columns four and five is entirely due to the way in which the internal pressure is obtained by each formulation, i.e., the thermodynamics in the bubble's interior. Note that the values of $T_{\max }$, $\boldsymbol{P}_{\max }$, and $\rho_{\max }$ predicted by the Prosperetti theory are lower when Flynn's radial equation is used instead of Keller-Miksis, suggesting that the additional compressibility term plays a significant role during the collapse. The values of $T_{\max }$ and $P_{\max }$ predicted by F-Prosperetti, however, are still higher than those predicted by Flynn by about $10 \%-20 \%$. The values of $\rho_{\max }$ are about the same in both cases. These results indicate that the Prosperetti theory does not allow as much heat to diffuse out into the liquid resulting in higher temperatures and pressures than the Flynn theory for the range of parameters considered here.

The phenomenon of sonoluminescence is primarily of thermal origin, i.e., caused by high temperatures rather than high pressures or densities. ${ }^{37}$ The determination of the minimum temperature required for light emission is, therefore, important in understanding the mechanisms involved. Since

TABLE II. Summary of maximum theoretical temperatures inside 15-to $20-\mu \mathrm{m}$ bubbles for the range of $P_{A}$ used during the experiments.

\begin{tabular}{lllll}
\hline \hline & $P_{A}$ (atm) & KM-Prosperetti & Flynn & F-Prosperetti \\
\hline Water & $(1.12-1.25)$ & $2500-7000 \mathrm{~K}$ & $2500-5000 \mathrm{~K}$ & $2500-6000 \mathrm{~K}$ \\
GLY42 & $(1.12-1.47)$ & $3000-10000 \mathrm{~K}$ & $2500-7000 \mathrm{~K}$ & $3000-8000 \mathrm{~K}$ \\
\hline \hline
\end{tabular}


TABLE III. Summary of maximum theoretical pressures inside 15- to $20-\mu \mathrm{m}$ bubbles for the range of $P_{A}$ used during the experiments.

\begin{tabular}{lllll}
\hline \hline & $P_{A}($ atm $)$ & KM-Prosperetti & Flynn & F-Prosperetti \\
\hline Water & $(1.12-1.25)$ & $1000-12000 \mathrm{~atm}$ & $2000-7000 \mathrm{~atm}$ & $2000-8000 \mathrm{~atm}$ \\
GLY42 & $(1.12-1.47)$ & $2000-40000 \mathrm{~atm}$ & $2000-22000 \mathrm{~atm}$ & $2000-20000 \mathrm{~atm}$ \\
\hline \hline
\end{tabular}

the acoustic pressure threshold for detectable light emission was observed in this study to be around $1.1 \mathrm{~atm}$, this indicates that, according to the theories, the minimum temperature necessary to generate observable SL is between 2000 and $3000 \mathrm{~K}$, corresponding to relative densities of about 100-200.

The most recent and precise measurement of temperatures inside cavitation bubbles is that by Suslick et al., ${ }^{2}$ using a comparative rate thermometry technique in aqueous solutions at $20 \mathrm{kHz}$. They measured a temperature of $5200 \pm 650 \mathrm{~K}$ at acoustic intensities of $24 \mathrm{~W} / \mathrm{cm}^{2}$, which corresponds to about $8 \mathrm{~atm}(800 \mathrm{kPa})$ assuming plane waves and ignoring the effect of the cavities. This temperature falls in the middle of the range of the theoretical temperatures calculated in this study, corresponding to $P_{A} \approx 1.3 \mathrm{~atm}$, much lower than the estimated 8 atm used in Suslick's experiment. In any case, the large number of bubbles present in the apparatus used by Suslick et al. would most likely result in the actual acoustic pressure being much less than that calculated using the plane-wave assumption.

As stated earlier, most experiments in which the temperature of collapsing cavities was measured probably required high-intensity sound fields that generated many cavities at once. In addition, transient cavitation was most likely the prevalent type of cavitation, implying that light emitting cavities did not last for more than a few acoustic periods. In the stable cavitation observed in this study, it has been determined that an increase in the acoustic pressure amplitude results in higher temperatures, as evidenced by the increase in the light emitted from the bubble. In a cavitation field where many bubbles are present, it is not obvious whether an increase in light emission is due to an increase in the amount of light emitted by each bubble, or an increase in the number of bubbles emitting light. Since the light emitted by each bubble should be proportional to the temperatures reached in its interior, it would be interesting to measure the dependence of the internal temperature on the acoustic intensity in experiments such as Suslick's.

The internal temperatures measured in Suslick's experiments, which were done at much higher pressure amplitudes, indicate that present theories of bubble pulsations may overestimate the internal temperature and possibly the

TABLE IV. Summary of maximum theoretical relative densities inside 15 to $20-\mu \mathrm{m}$ bubbles for the range of $P_{A}$ used during the experiments.

\begin{tabular}{lcccc}
\hline \hline & $P_{A}$ (atm) & KM-Prosperetti & Flynn & F-Prosperetti \\
\hline Water & $(1.12-1.25)$ & $150-500$ & $200-400$ & $100-400$ \\
GLY42 & $(1.12-1.47)$ & $200-1300$ & $150-700$ & $200-700$ \\
\hline \hline
\end{tabular}

internal pressure too. This overestimation may be explained by the failure of the assumptions made in the models. Specifically, the dissociation of the gas molecules due to the high temperatures attained would increase the number density and, therefore, the internal (gas) pressure, arresting the bubble collapse sooner than predicted. When temperatures of $5000 \mathrm{~K}$ or higher are reached, it is likely that the energy of the collapse is partitioned into other chemical processes, instead of increasing the temperature of the gas. Some of these processes include dissociation of the gas molecules and ionization. Thus, as the strength of the collapse increases, i.e., at larger values of $P_{A}$, the internal temperature is expected to reach a plateau. In addition, the models underestimate the effects of energy dissipation due to the compressibility of the liquid. A compressible liquid results in the formation of shock waves that may carry a significant portion of the collapse energy away from the bubble.

\section{Phase of sonoluminescence}

Using the light scattering apparatus and the photomultiplier (PMT) SL detection system shown in Fig. 7(a) and (b), the phase of SL was measured relative to the instantaneous radius of the bubble. This is the most direct way of determining the point during the bubble motion in which the SL flash is emitted. Since both the photomultiplier tube (PMT) and the laser could not be engaged simultaneously, an intermediate phase reference, the side pill transducer, had to be used.

The procedure was as follows: After positioning a pulsating bubble in the center of the laser beam, a scattered-light intensity versus time trace was obtained simultaneously with the pill output and subsequently stored in the computer. The laser and the room lights were then turned off and the PMT activated. Similarly, simultaneous traces of the PMT output and the pill transducer output were stored in the computer. Several traces were obtained within a few seconds of each other to verify that experimental conditions remained as identical as possible. The correlation between the two traces was determined graphically by overlapping the two pill transducer traces until a best fit was obtained. Figure 18 is a plot of the experimental results clearly showing that SL is produced during the collapse of the bubble. The error in this measurement was estimated to be $\pm 5 \mathrm{deg}$. This result is significant because it dramatically illustrates the causal relationship between the bubble collapse and the light emission.

\section{Sonoluminescence experiments}

After the simultaneity of the light emission and the collapse of the bubble was established, the phase of the SL emission was used to study the behavior and the time evolution of 


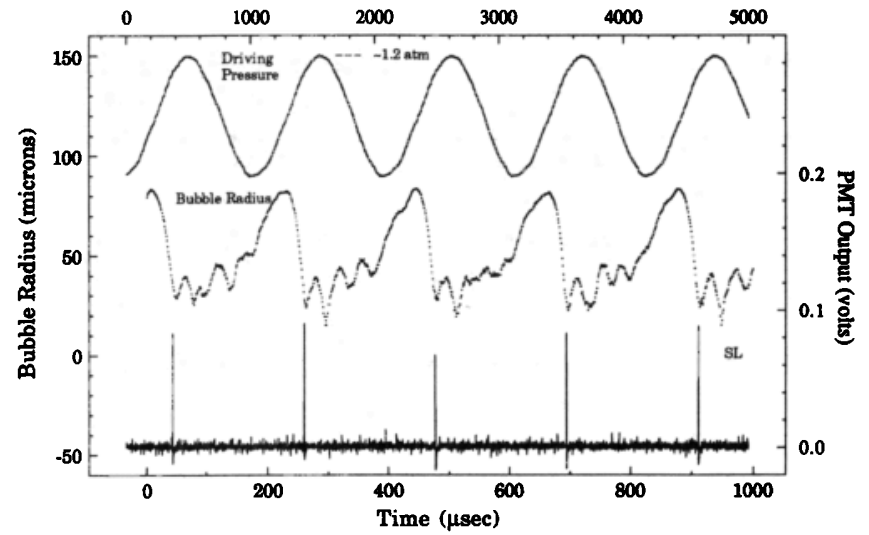

FIG. 18. Simultaneous plots of the sound field (top), bubble radius (middle) and sonoluminescence (bottom) in GLY21 at $P_{A}=1.2 \mathrm{~atm}$ and $f=22.3 \mathrm{kHz}$.

cavitation bubbles as they interacted with the sound field and with each other. This was done by measuring the phase of the light emitted by the bubbles to obtain the phase of the bubble collapse. In view of the already established coincidence of the light emission and the bubble collapse, these two terms will be used interchangeably.

The time-to-amplitude converter system provided a very efficient method of recording the phase of SL for thousands of cycles and in real-time if desired. The phase of SL was measured for single bubbles using the different liquid mixtures. Measurements were also made of the phase of SL during streamer activity when the cavitation field was composed of many bubbles sonoluminescing simultaneously. The purpose of this latter experiment was to study the behavior of bubbles during intense cavitation activity. To interpret the observations made in multibubble cavitation fields, the results for a single bubble will be discussed first.

\section{Single bubble cavitation field}

Figure 19 shows the phase of SL at approximately 1.1 atm in water, plotted as a function of time in units of acoustic periods. In this data set, a total 2000 data points were taken corresponding to approximately $0.1 \mathrm{~s}$. The small oscillations on these data are due to $60-\mathrm{Hz}$ line noise that could not be easily filtered. Typical $60-\mathrm{Hz}$ noise levels were $\pm 1 \mathrm{deg}$. This is an example of the phase of the light emission for a single bubble pulsating radially.

If one ignores the $60-\mathrm{Hz}$ noise, these data demonstrate a great stability of this dynamic system. Even though the bubble grows to several times its equilibrium size and then collapses within microseconds with such a violence that it emits optical energy, it still repeats this process for thousands of cycles with a regularity more precise than was possible to measure. Indeed, Barber and Putterman ${ }^{38,39}$ have determined that the "jitter" in the repeatability of this process is less that 50 ps. Furthermore, the duration of the SL flash was so short that we were also unable to resolve its length with the available instruments. Subsequently, Putterman and his colleagues have obtained photomultiplier tubes with increasingly fast response times and have demonstrated that the SL pulse duration is also less that $50 \mathrm{ps}^{38,39}$ These latter

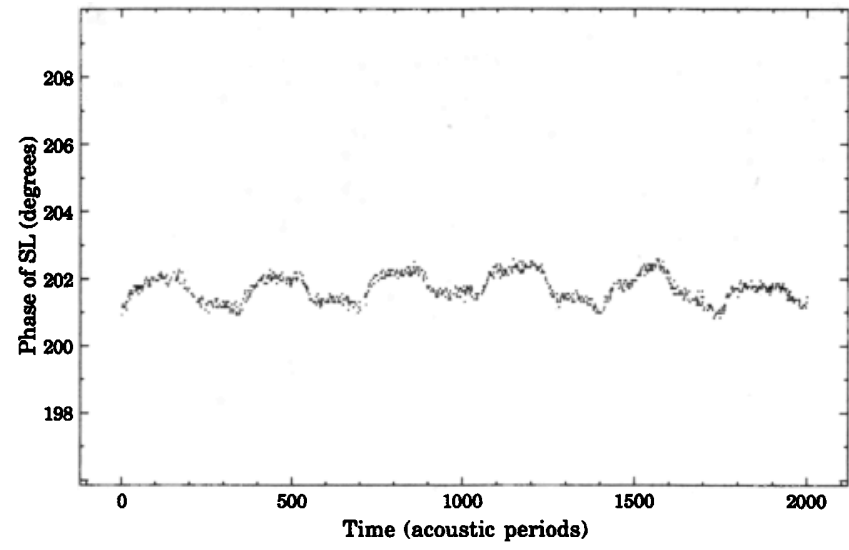

FIG. 19. Phase of sonoluminescence emitted by a single bubble as a function of time. It was measured in water at $P_{A} \approx 1.1 \mathrm{~atm}$ and $f=21.0 \mathrm{kHz}$ ( $T=47.6 \mu \mathrm{s}$ ) by the time-to-amplitude converter system. The oscillation in the signal is due primarily to $60-\mathrm{Hz}$ noise.

experiments demonstrate the remarkable nature of this phenomenon, suggesting that a major part of it is still not clearly understood.

To determine if a single bubble was present, the output of the side pill transducer was monitored. This output would become noticeably irregular and noisy when streamers were present. For a single bubble, the signal was symmetrical except for a small notch, probably due to the shock wave produced by the bubble collapse. For the case shown in Fig. 19, the value of the phase was $\sim 201 \mathrm{deg}$. A range of values was measured between 190 and $220 \mathrm{deg}$ for pressure amplitudes between 1.0 and $1.4 \mathrm{~atm}$ in water.

At the high end of the pressure amplitude range, the levitated bubble was occasionally observed to become unstable for a fraction of a second, after which stability was restored. Following this transition, the bubble's position shifted in the vertical direction. This new position was always away from the antinode. When the light emission was monitored, it was observed that the intensity of each flash was sharply decreased after the transition had occurred. The phase of the emissions was also decreased. These observations are illustrated in Fig. 20. The phase can be clearly seen

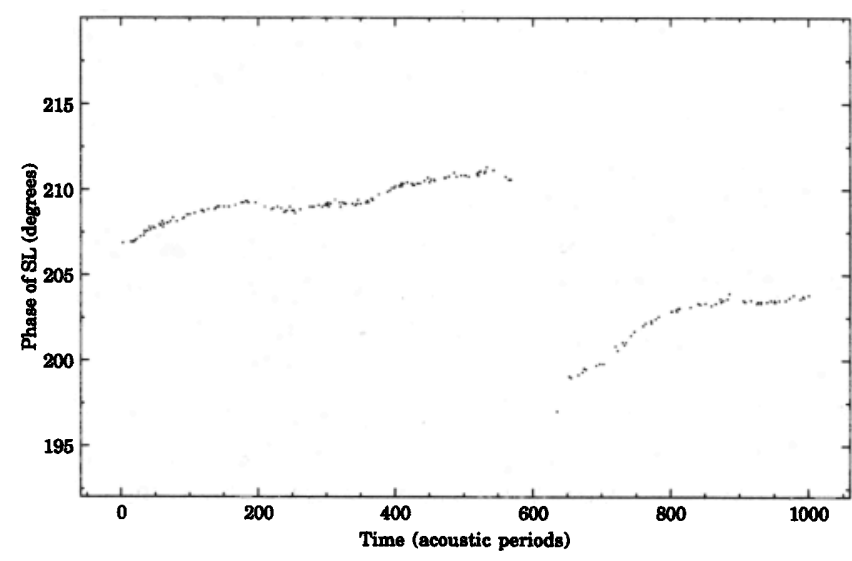

FIG. 20. Phase of sonoluminescence emitted by a single bubble as a function of time. It was measured in water at $P_{A} \approx 1.2 \mathrm{~atm}$ and $f=21.0 \mathrm{kHz}$ $(T=47.6 \mu \mathrm{s})$ by the time-to-amplitude converter system during a transition (see text). 
to decrease sharply during the transition at 600 cycles. One explanation for this decrease of the phase is that there is a decrease in the equilibrium bubble radius, i.e., a breakup of the bubble due to surface instabilities. This type of breakup usually occurs via the ejection of microbubbles. ${ }^{4,40}$ After reducing its size, the bubble regains its stability due to the larger surface-tension pressure $\left(2 \sigma / R_{0}\right)$. This occasional, and sometimes periodic, breakup can be understood in terms of the bubble response curve as a function of equilibrium radius (see Fig. 5). As the bubble grows by rectified diffusion, resonances are encountered and the bubble response increases rapidly. If the surface instability threshold happens to be below one of these peaks, breakup will occur and the bubble size is quickly reduced. This cycle may repeat depending on $P_{A}$ and the new value of $R_{0}$, among other parameters. In addition, by plotting the pulsation amplitude, $R_{\max }$, and the phase of the collapse as a function of $R_{0}$, a direct correlation between these two quantities can be established. When $R_{\max }$ increases (decreases) the phase also increases (decreases), as shown in Fig. 5 . Note that a change in the radius $R_{0}$ as small as $1 \mu \mathrm{m}$ can result in the phase of collapse changing by as much as $20 \mathrm{deg}$, depending on the bubble size. Thus, it does not require a large change in radius to effect a large change in the phase.

It appears that before the bubble regains stability, its response amplitude or, equivalently, equilibrium size must be decreased. This is not surprising since instabilities in the gas-liquid interface are triggered by large accelerations. ${ }^{41}$ This observation can be explained as follows: After developing instabilities, the bubble sheds microbubbles, reducing its size and its response amplitude. Both effects increase the stability of the bubble and the pulsations become spherically symmetric again. The bubble then grows slowly by rectified diffusion, sheds microbubbles, etc. This behavior is commonly seen in single bubbles at the higher pressure amplitudes $(\sim 1.3-1.4 \mathrm{~atm})$.

At the higher end of the pressure range, instabilities occurred repeatedly with a period of a few seconds. At the higher end, transitions often occurred rapidly and consecutively as shown in Fig. 21. Here, it may be postulated that the

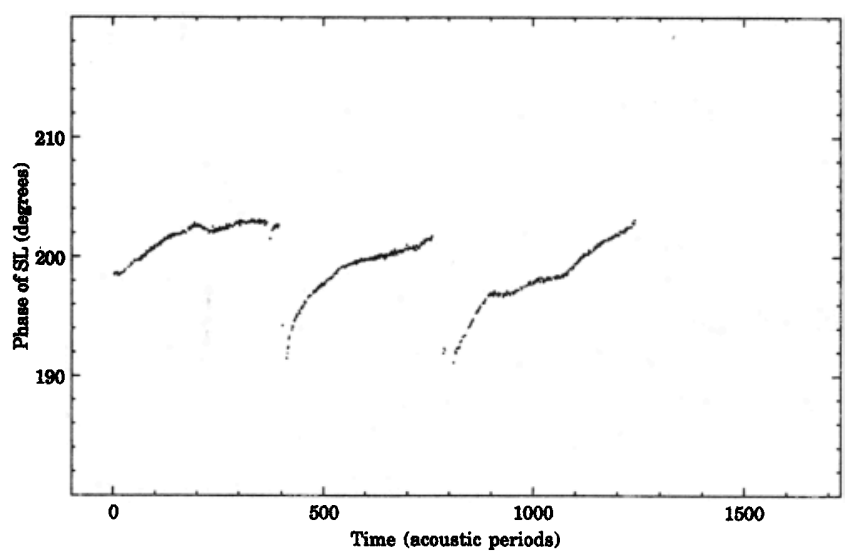

FIG. 21. Phase of sonoluminescence emitted by a single bubble as a function of time. It was measured in water at $P_{A} \approx 1.3$ atm and $f=21.0 \mathrm{kHz}$ $(T=47.6 \mu \mathrm{s})$ by the time-to-amplitude converter system during buibble breakup and disappearance. pressure amplitude was high enough that surface waves were easily excited, and the bubble was unable to regain its stability before disintegrating. If the residual microbubbles are large enough or close enough to the initiating bubble, coalescence may occur before they can dissolve. This process may explain the rapid bubble growth inferred from the rapid increase in the phase of collapse shown in Fig. 21. Sometimes the bubble disappeared unexpectedly, as occurred here when the bubble suddenly stopped emitting light around cycle 1250. This bubble disappearance also can be observed with the unaided eye or through the microscope. A similar type of bubble annihilation was reported by Nyborg and Hughes ${ }^{4}$ during observation of cavitation on the surface of a vibrating bar driven at $20 \mathrm{kHz}$. Using high-speed photography, they reported bubbles that disappeared in less than six acoustic periods. An explanation similar to ours was given by Nyborg and Hughes ${ }^{4}$ for this phenomenon.

In summary, it appears that bubbles grow and breakup in a periodic or quasiperiodic fashion during radial pulsations. A breakup is usually reflected by a decrease in the bubble response and the phase of the collapse. Bubble growth can occur by coalescence or rectified diffusion and can be detected by an increase of the response and the phase of the bubble collapse.

\section{Multibubble cavitation fields}

In this section, the observations made from a cavitation zone composed of streamers will be discussed. High-speed photographs taken during the experiments revealed that these streamers consisted of fast-moving bubbles that were undergoing rapid fission and fusion. An example of the measurement of the phase of collapse in water at around $1.3 \mathrm{~atm}$ is shown in Fig. 22. In this data set, the phase of collapse appears to change irregularly between 185 and $215 \mathrm{deg}$. Most of the measurements made of the collapse phase in multibubble cavitation fields were in this range. This range coincided with the collapse phases measured for single bubbles, which were determined to be between 15 and $20 \mu \mathrm{m}$ in radius. It thus appears that in a cavitation field at $21 \mathrm{kHz}$, bubbles that emit SL are in the range $15 \leqslant R_{0} \leqslant 20 \mu \mathrm{m}$. This

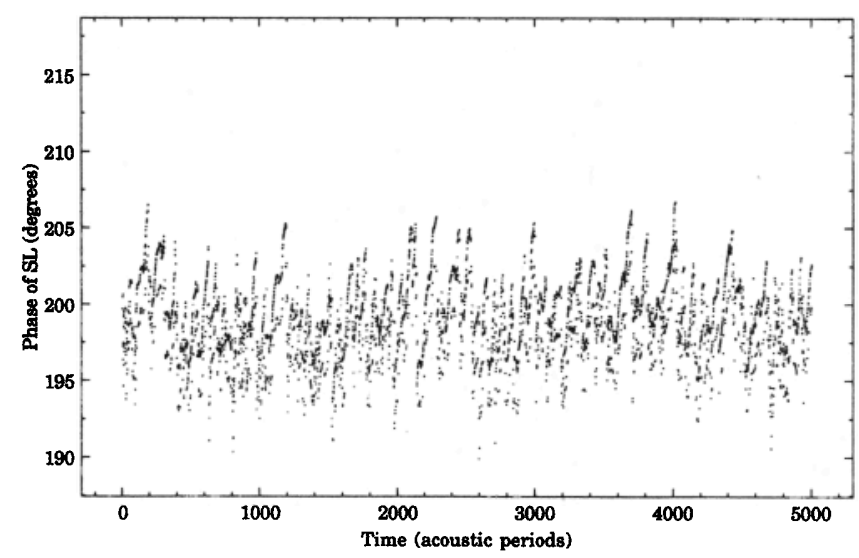

FIG. 22. Phase of sonoluminescence emitted by a multibubble cavitation field as a function of time. It was measured in water at $P_{A} \approx 1.3 \mathrm{~atm}$ and $f=21.0 \mathrm{kHz}(T=47.6 \mu \mathrm{s})$ by the time-to-amplitude converter system. 
range of bubble sizes may be even smaller since some scatter in the measured values of the phase may be due to fluctuations of the driving pressure. Also, the fact that the range of values for the phase of collapse of single bubbles and cavitation field bubbles coincide may be an indication that only bubbles pulsating radially emit SL. Evidence for this hypothesis is given by our observations that when a single bubble undergoes surface oscillations, the SL output decreases by an order of magnitude. It was not uncommon to observe a single bubble pulsating radially and emitting SL flashes every cycle for as many as 1000 consecutive cycles. When surface waves were excited, as evidenced by dancing motion, the average number of SL flashes was about one in ten cycles. This seems to imply that a spherical collapse is necessary to generate sufficiently high temperatures and pressures in the interior of the bubble.

In addition, the data displayed in Fig. 23 reveal the same type of cyclic behavior observed in a single bubble as described in the previous section. This behavior was observed often in a multibubble cavitation field, although it was not always repeatable. It was usually observed at the higher pressure amplitudes (1.3-1.5 atm). Nevertheless, these data show that the same cyclic behavior observed for a single bubble also occurs in a multibubble cavitation field. Similarly, this behavior can be attributed to the fission of bubbles by surface instabilities followed by coalescence or rectified diffusion.

In summary, we have observed cyclic behavior in both single and multibubble cavitation fields. This behavior has been observed before by other investigators and can be caused by resonances of the bubble motion encountered as the bubble grows. These resonances trigger surface instabilities that result in a reduction of the bubble size due to fission. As the bubbles regrow by rectified diffusion or coalescence, the process repeats itself. In addition, the coincidence of the phase of collapse for both single and multibubble cavitation suggests that spherical collapses are required to generate the high temperatures and pressures responsible for most cavitation-related effects. It has also been demonstrated that the phase of SL emission can be used to study the behavior of

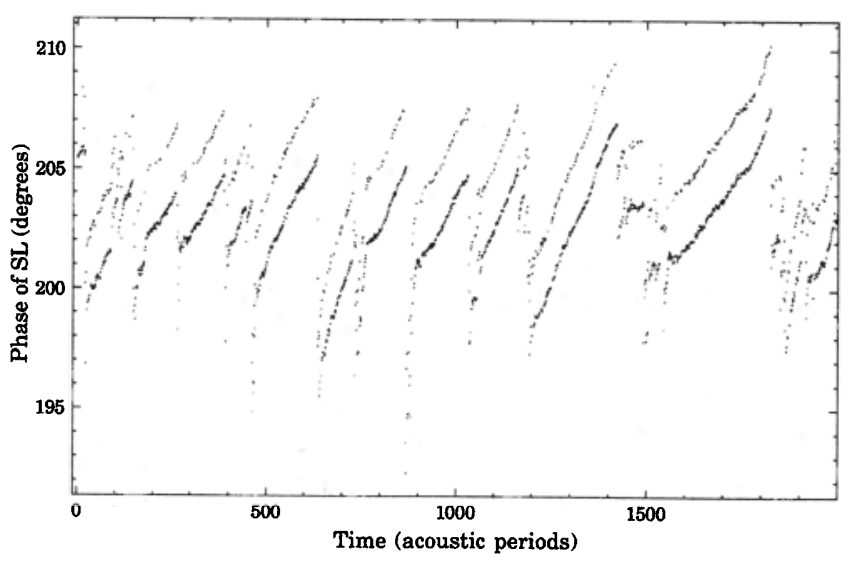

FIG. 23. Phase of sonoluminescence emitted by a multibubble cavitation field as a function of time. It was measured in water at $P_{A} \approx 1.3 \mathrm{~atm}$ and $f=21.0 \mathrm{kHz}(T=47.6 \mu \mathrm{s})$ by the time-to-amplitude converter system. bubbles in cavitation fields on a time scale on the order of an acoustic period.

\section{SUMMARY AND CONCLUSIONS}

(1) A previously undiscovered region of stability exists in the pressure-radius parameter space above the level of both surface instability and the threshold for rectified diffusion thresholds for radially pulsating bubbles. The mechanisms through which this stability is attained are not yet understood. The observations reported here indicate the existence of a transient cavitation threshold, which forms an upper boundary to this stable region. Above this threshold, spherical stability was never attained. It is believed that the rapid bubble collapse promotes the formation and enhances the magnitude of surface instabilities that cause the bubble to disintegrate.

The phase of the light emission was observed for thousands of acoustic periods to monitor the long-term behavior of bubbles driven at moderate intensities in the cavitation field. From these observations, it was concluded that, except in the small region of stability, bubbles grow by rectified diffusion until surface waves cause them to fission. These two phenomena affect the periodic pulsations of the bubbles so that they exhibit a "life cycle." This life cycle has a relatively slow growth followed by an abrupt fragmentation and has a period on the order of $10 \mathrm{~ms}$ ( $\sim 200$ acoustic periods), depending on the driving amplitude.

(2) Experimental radius-time curves for single bubbles pulsating radially at large amplitudes have been obtained. These bubbles were observed in water/glycerine mixtures at pressure amplitudes between 1.1 and $1.5 \mathrm{~atm}$ at $20-25 \mathrm{kHz}$. The pulsation amplitude, the phase of the collapse and the number of minima have been measured for a range of pressure amplitudes and bubble sizes.

(3) Sonoluminescence from stable cavitation at $20 \mathrm{kHz}$ is most effectively emitted by bubbles in the size range 15-20 $\mu \mathrm{m}$ rather than by bubbles resonating at their natural frequency. These bubbles are small enough so that, although pulsating at large amplitudes, they remain spherically symmetric, rapidly compressing the gas and heating it to high temperatures.

(4) The applicability of three theoretical formulations of bubble dynamics has been evaluated in a range of acoustic pressure amplitudes near the threshold for sonoluminescence. General agreement was found between the experimental and theoretical values of the pulsation amplitude, phase of collapse and the number of rebounds. The number of rebounds was observed to be lower and the amplitude of each rebound larger in amplitude than expected from the theories. The data suggest that the physical conditions attained during the collapse may be outside the limits of the theories.

(5) From the theoretical calculations made for the parameters measured at the threshold for light emission, it is estimated that internal temperatures in the range 2000-3000 $\mathrm{K}$ are necessary to produce detectable sonoluminescence. This finding supports the Chemiluminescent model as the mechanism for light production. 


\section{ACKNOWLEDGMENTS}

The authors wish to acknowledge many helpful discussions with Andrea Prosperetti, S. Putterman, K. Suslick, and the financial support of the Office of Naval Research.

${ }^{1}$ T. K. Saksena and W. L. Nyborg, "Sonoluminescence from stable cavitation," J. Chem. Phys. 53, 1722 (1970).

${ }^{2}$ K. S. Suslick, D. A. Hammerton, and R. E. Cline, "The sonochemical hot spot," Am. Chem. Soc. 108, 5641-5642 (1986).

${ }^{3}$ L. A. Crum and G. T. Reynolds, "Sonoluminescence produced by stable cavitation," J. Acoust. Soc. Am. 78, 137-139 (1985).

${ }^{4}$ W. Nyborg and D. E. Hughes, "Bubble annihilation in cavitation streamers," J. Acoust. Soc. Am. 42, 891 (1967).

${ }^{5}$ F. G. Blake, "The onset of cavitation in liquids," Tech. Memo. No. 12, Harvard Acoustics Research Laboratory (1949).

${ }^{6} \mathrm{G}$. W. Willard, "Ultrasonically induced cavitation in water: a step-by-step process," J. Acoust. Soc. Am. 25, 669 (1953).

${ }^{7}$ H. G. Flynn, Physical Acoustics, edited by W. P. Mason (Academic, New York, 1964), Vol. 1, part B.

${ }^{8}$ H. G. Flynn, "Cavitation dynamics. II. Free pulsations and models for cavitation bubbles," J. Acoust. Soc. Am. 58, 1160 (1975).

${ }^{9}$ N. Marinesco and J. J. Trillat, C. R. Acad. Sci. Paris 196, 858 (1933).

${ }^{10}$ P. Paounoff, C. R. Hebd. Seances Acad. Sci. Paris 44, 261 (1947).

"P. Gunther, E. Heim, A. Schmitt, and W. Zeil, Z. Naturforsch. 12A, 521 (1957a).

${ }^{12}$ W. U. Wagner, Z. Angew. Phys. 10, 445 (1958).

${ }^{13} \mathrm{~K}$. Negishi, Acustica 10, 124 (1960).

${ }^{14}$ R. Q. Macleay and L. V. Holroyd, "Space-time analysis of the sonoluminescence emitted by cavitated water," J. Appl. Phys. 32, 449-453 (1961).

${ }^{15} \mathrm{P}$. Jarman and K. J. Taylor. "The timing of the main and secondary flashes of sonoluminescence from acoustically cavitated water," Acustica 23, 243 (1970).

${ }^{16}$ B. E. Noltingk and E. A. Neppiras. "Cavitation produced by ultrasonics," Proc. Phys. Soc. London Sect. B 63, 674 (1950).

${ }^{17} \mathrm{~J}$. B. Keller and M. Miksis, "Bubble oscillations of large amplitude," J. Acoust. Soc. Am. 68, 628 (1980).

${ }^{18}$ A. Prosperetti, L. A. Crum, and K. W. Commander, "Nonlinear bubble dynamics," J. Acoust. Soc. Am. 83, 502 (1986).

${ }^{19}$ H. G. Flynn, "Cavitation dynamics. I. A Mathematical formulation," J. Acoust. Soc. Am. 57, 1379 (1975).

${ }^{20}$ A. Prosperetti, "Acoustic cavitation series part two-Bubble phenomena in sound fields: part one," Ultrasonics 22, 69 (1984).
${ }^{21}$ A. Prosperetti, "Nonlinear oscillations of gas bubbles in liquids: steadystate solutions," J. Acoust. Soc. Am. 56, 878 (1974).

${ }^{22} \mathrm{~W}$. Lauterborn, "Numerical investigation of nonlinear oscillations of gas bubbles in liquids," J. Acoust. Soc. Am. 59, 283-293 (1976).

${ }^{23}$ L. A. Crum and A. Prosperetti, "Erratum and comments on 'Nonlinear oscillations of gas bubbles in liquids: An interpretation of some experimental results'," J. Acoust. Soc. Am. 75, 1910 (1984).

${ }^{24} \mathrm{D}$. F. Gaitan, "An experimental investigation of acoustic cavitation in gaseous liquids," Ph. D. dissertation, University of Mississippi (1990).

${ }^{25}$ IMSL, Mathematical Software Library (IMSL, Houston, 1987).

${ }^{26} \mathrm{C}$. W. Gear, Numerical Initial-Value Problems in Ordinary Differential Equations (Prentice-Hall, Englewood Cliffs, NJ, 1971).

${ }^{27}$ V. Kamath and A. Prosperetti, "Numerical integration methods in gasbubble dynamics," J. Acoust. Soc. Am. 85, 1538 (1989).

${ }^{28} \mathrm{~W}$. Lauterborn and U. Parlitz, "Methods of chaos physics and their applications to acoustics," J. Acoust. Soc. Am. 84, 1975-1993 (1988).

${ }^{29} \mathrm{~L}$. A. Crum, "The acoustic radiation pressure on a liquid droplet in a stationary sound field," Tech. Report, U.S. Naval Academy (1970).

${ }^{30} \mathrm{G}$. M. Hansen, "Mie scattering as a technique for the sizing of air bubbles," Ph. D. dissertation, University of Mississippi (1984).

${ }^{31}$ R. G. Holt, "Experimental observations of the nonlinear response of single bubbles to an applied acoustic field," $P$ h. D. dissertation, University of Mississippi (1988).

${ }^{32}$ S. D. Horsburgh, "Radial instabilities of a pulsating air bubble in water," Ph. D. dissertation, University of Mississippi (1990).

${ }^{33}$ R. K. Gould, "Simple method for calibrating small omnidirectional hydrophone," J. Acoust. Soc. Am. 43, 1185 (1968).

${ }^{34} \mathrm{~W}$. Lauterborn, "Cavitation dynamics-new tools for an intricate problem," Appl. Sci. Res. 38, 165-178 (1982).

${ }^{35}$ L. A. Crum, "Bjerknes forces on bubbles in a stationary sound field," J. Acoust. Soc. Am. 57, 1363 (1975).

${ }^{36} \mathrm{~A}$. Eller and G. H. Flynn, "Rectified diffusion during nonlinear pulsations of cavitation bubbles," J. Acoust. Soc. Am. 37, 493 (1965).

${ }^{37}$ C. Sehgal, R. P. Steer, R. G. Sutherland, and R. E. Verral, "Sonoluminescence of argon saturated alkali metal salt solutions as a probe of acoustic cavitation," J. Chem. Phys. 70, 2242-2248 (1979).

${ }^{38}$ B. P. Barber and S. J. Putterman, "Observations of synchronous picosecond sonoluminescence," Nature 352, 318 (1991).

${ }^{39}$ B. P. Barber, R. Hiller, K. Arisaka, H. Fetterman, and S. J. Putterman, "Resolving the picosecond characteristics of synchronous sonoluminescence," submitted to J. Acoust. Soc. Am.

${ }^{40}$ E. Neppiras and E. E. Fill, "A cyclic cavitation process," J. Acoust. Soc. Am. 46, 1264 (1969).

${ }^{41}$ D. Y. Hsieh, "On the thresholds for surface waves and subharmonics of an oscillating bubbles," J. Acoust. Soc. Am. 56, 392 (1974). 